\title{
Probing the Wave Nature of Light-Matter Interaction
}

\author{
D. E. Boone1, C. H. Jackson², A. T. Swecker ${ }^{2}$, J. S. Hergenrather', K. S. Wenger², O. Kokhan³, \\ B. Terzić4, I. Melnikov², I. N. Ivanov5, E. C. Stevens ${ }^{6}$, G. Scarel ${ }^{2 *}$
}

${ }^{1}$ College of Integrated Science and Engineering, James Madison University, Harrisonburg, Virginia, USA

${ }^{2}$ Department of Physics and Astronomy, James Madison University, Harrisonburg, VA, USA

${ }^{3}$ Department of Chemistry and Biochemistry, James Madison University, Harrisonburg, VA, USA

${ }^{4}$ Department of Physics, Old Dominion University, Norfolk, VA, USA

${ }^{5}$ Center for Nanophase Materials Sciences, Oak Ridge National Laboratory, Oak Ridge, TN, USA

${ }^{6}$ Department of Chemical and Biomolecular Engineering, North Carolina State University, Centennial Campus, Raleigh, NC, USA

Email: *scarelgx@jmu.edu

How to cite this paper: Boone, D.E., Jackson, C.H., Swecker, A.T., Hergenrather, J.S., Wenger, K.S., Kokhan, O., Terzić, B., Melnikov, I., Ivanov, I.N., Stevens, E.C. and Scarel, G. (2018) Probing the Wave Nature of Light-Matter Interaction. World Journal of Condensed Matter Physics, 8, 62-89. https://doi.org/10.4236/wjcmp.2018.82005

Received: May 5, 2018

Accepted: May 27, 2018

Published: May 30, 2018

Copyright (c) 2018 by authors and Scientific Research Publishing Inc. This work is licensed under the Creative Commons Attribution International License (CC BY 4.0).

http://creativecommons.org/licenses/by/4.0/

\section{Abstract}

The wave-particle duality of light is a controversial topic in modern physics. In this context, this work highlights the ability of the wave-nature of light on its own to account for the conservation of energy in light-matter interaction. Two simple fundamental properties of light as wave are involved: its period and its power P. The power P depends only on the amplitude of the wave's electric and magnetic fields (Poynting's vector), and can easily be measured with a power sensor for visible and infrared lasers. The advantage of such a wave-based approach is that it unveils unexpected effects of light's power $\mathrm{P}$ capable of explaining numerous results published in current scientific literature, of correlating phenomena otherwise considered as disjointed, and of making predictions on ways to employ the electromagnetic (EM) waves which so far are unexplored. In this framework, this work focuses on determining the magnitude of the time interval that, coupled with light's power $\mathrm{P}$, establishes the energy conserved in the exchange of energy between light and matter. To reach this goal, capacitors were excited with visible and IR lasers at variable average power P. As the result of combining experimental measurements and simulations based on the law of conservation of energy, it was found that the product of the period of the light by its power $\mathrm{P}$ fixes the magnitude of the energy conserved in light's interaction with the capacitors. This finding highlights that the energy exchanged is defined in the time interval equal to the period of the light's wave. The validity of the finding is shown to hold in light's interaction with matter in general, e.g. in the photoelectric effect with $\mathrm{x}$-rays, in the transfer of electrons between energy levels in semiconducting interfaces of 
field effect transistors, in the activation of photosynthetic reactions, and in the generation of action potentials in retinal ganglion cells to enable vision in vertebrates. Finally, the validity of the finding is investigated in the low frequency spectrum of the EM waves by exploring possible consequences in microwave technology, and in harvesting through capacitors the radio waves dispersed in the environment after being used in telecommunications as a source of usable electricity.

\section{Keywords}

Infrared, Light-Matter Interaction, Conservation Of Energy, Wave Energy Harvesting

\section{Introduction}

In the current debate on wave-particle duality of light and its role in light-matter interaction, two questions rise: 1) are photons necessary and sufficient participants? and 2) what decides the magnitude of the phenomena arising from light-matter interaction? We address these questions in our study of conservation of energy in light-matter interaction.

Photons are particles of light, which provide a beautiful example of conservation of energy through the photoelectric effect. Ultraviolet (UV) and x-ray photons with frequency $v_{p h}$ and energy $E_{p h}=h v_{p h}$, where $h$ is Planck's constant $\left(6.63 \times 10^{-34} \mathrm{Js}\right)$, can eject an electron out of an atom such that $h v_{p h}$ $=E_{b}+E_{k}+\varphi$, where $E_{b}$ is the binding energy of the electron in the atom, $E_{k}$ the kinetic energy of the electron after being ejected from the atom by the photon, and $\phi$ a work function. For example, an x-ray photon with energy $E_{x}=$ $1.2536 \mathrm{keV}$ produced by a $\mathrm{Mg} \mathrm{K} \alpha$ source can extract an electron with $E_{k}=964.2$ $\mathrm{eV}$ from a carbon atom to which the electron was bound with $E_{b}=285.4 \mathrm{eV}$ and $\phi=4 \mathrm{eV}$ [1] [2]. Thus, in the photoelectric effect, the conserved energy is $E_{p h}=h v_{p h}$. Once the photon interacts with matter, this energy distributes itself into various components: one component of magnitude $E_{b}$ unbinds the electron from the atom, another one of magnitude $\phi$ unbinds the electron from the material, and the last component transfers into the kinetic energy $E_{k}$ of the free electron. This concept is successfully exploited in $\mathrm{x}$-ray photoelectron spectroscopy (XPS) [3] [4]. However, as the photon's frequency $v_{p h}$ decreases below that of the UV light, the energy of one single photon becomes small and less than the magnitude conserved in the interaction between light and matter. Such a mismatch is observed, for example, in electronic devices where photons are used to generate a photocurrent by providing energy to electrons to jump over an energy barrier. To overcome a $0.32 \mathrm{eV}$ barrier, a photon with frequency $v=77.2 \mathrm{GHz}$ (far infrared (IR)) would suffice according to $E_{p h}=h v=0.32 \mathrm{eV}$. Even though $0.32 \mathrm{eV}$ is only $1 / 4$ of the energy of a photon with frequency $v=0.3 \mathrm{PHz}$, Barati et al. [5] found that near IR light at $v=0.3 \mathrm{PHz}$ and average power $P=17 \mu \mathrm{W}$ 
can excite the electrons over the $0.32 \mathrm{eV}$ gap at the $\mathrm{MoSe}_{2} / 2 L$-WSe $\mathrm{W}_{2}$ interface in heterostructure photocells between the conduction band (CB) of $\operatorname{MoSe}_{2}\left(E_{C B M o}\right)$ and the $\mathrm{CB}$ of $\mathrm{WSe}_{2}\left(E_{C B W}\right)$. Similarly, Adinolfi et al. [6], report that near IR light at $v=0.23 \mathrm{PHz}$ with $P$ in the $n W$ range, or below, maximizes the photocurrent production in silicon-based photovoltage field effect transistors (FETs). Sarker et al. [7] confirm the trend by showing that green light at $v=0.56 \mathrm{PHz}$ with $P=5 \mu \mathrm{W}$ generates photoexcited carriers by triggering electrons to jump from in-gap impurity levels to the $\mathrm{CB}$ in $4 H$-SiC used as substrate in graphene FETs. These results suggest that the average power of the electromagnetic (EM) wave might play a role in photocurrent generation.

The energy needed in photosynthetic processes also challenges the amount of energy that single photons can provide. Photosynthetic reactions are triggered by solar light at an average power per unit area $P \approx 136 \frac{\mathrm{mW}}{\mathrm{cm}^{2}}$ [8] [9]. Typical activation energies per molecule are, e.g., $0.5 \mathrm{eV}$ for the oxidation of cytochrome [10], or $0.72 \mathrm{eV}$ for the $\mathrm{CO}_{2}$ exchange [11], or $1.6 \mathrm{eV}$ for the $\mathrm{CO}_{2}$ oxidation [12]. A careful analysis of the light's wavelengths in and out of a photosynthetic organism shows that, e.g., in the carotenoid $\mathrm{S}_{2}$ stimulated emission [13] with green light in at $\lambda_{\text {in }}=520 \mathrm{~nm} \quad\left(v_{\text {in }}=0.58 \mathrm{PHz}\right)$ and green light out at $\lambda_{\text {out }}=540 \mathrm{~nm}$ $\left(v_{\text {out }}=0.55 \mathrm{PHz}\right)$, the exchanged energy is $\left|h\left(v_{\text {out }}-v_{\text {in }}\right)\right| \approx 0.12 \mathrm{eV}$. In the bacteriochlorophylls-a (BChl-a) $\mathrm{Q}_{\mathrm{x}}$ state, the same amount of energy of $\approx 0.12 \mathrm{eV}$ is exchanged when green light in at $\lambda_{\text {in }}=540 \mathrm{~nm}\left(v_{\text {in }}=0.55 \mathrm{PHz}\right)$ releases yellow light at $\lambda_{\text {out }}=580 \mathrm{~nm} \quad\left(v_{\text {out }}=0.52 \mathrm{PHz}\right)$. We estimate that $\sim 4,6$, and 14 couples of photons are required to provide the $0.5 \mathrm{eV}, 0.72 \mathrm{eV}$, and $1.6 \mathrm{eV}$ activation energies per molecule to initiate the photosynthetic reactions mentioned above. Here we raise the question whether the average power per unit area $P \approx 136 \frac{\mathrm{mW}}{\mathrm{cm}^{2}}$ of the solar light might play a role in providing a wider range and higher values of the energy exchanged between solar light and photosynthetic organisms.

Another process that challenges the amount of energy that single photons can provide is the energy conversion in retinal ganglion cells (RGCs) to enable vision in vertebrates. The RGCs act as capacitors with baseline capacitance of the order of few $\mathrm{pF}$ [14]. When illuminated by solar light, the RGCs elicit an action potential $V_{a p} \approx 70 \mathrm{mV}$ while simultaneously altering the capacitance $C$ by $\approx 0.2 \mathrm{pF}$ [14]. The assumption that the energy of one photon contributes to the production of $V_{a p}$ leads to $h v_{p h}=\frac{1}{2} C V_{a p}^{2}$. This equation requires one photon with $v_{p h}=0.74 \times 10^{18} \mathrm{~Hz}$, which is in the $\mathrm{x}$-ray region of the EM spectrum! Alternatively, we can hypothesize that the process is enabled by 1345 green light photons at $\lambda=540 \mathrm{~nm}(v=0.55 \mathrm{PHz})$. However, we can question whether there is a link between these 1345 photons and the average power per unit area $P \approx 136 \frac{\mathrm{mW}}{\mathrm{cm}^{2}}$ of the solar light in enabling visible light to elicit the action potential in the RGCs. 
In this work, we exploit the wave nature of light to address the mismatch between the low frequency photon's energy and the energy conserved in the exchange of energy between light and devices or natural light harvesting (LH) systems. We start off by recalling that light is an EM wave whose power $P$ per unit area is the modulus of Poynting's vector, $|\boldsymbol{S}|=|\boldsymbol{E} \times \boldsymbol{H}|=\frac{P}{A}$, where $A$ is area, $\boldsymbol{E}$ the electric field, and $\boldsymbol{H}$ the magnetic field [15]. Experimentally, the average power $P$ of visible and IR lasers is easily measured with power sensors. We then hypothesize that the energy conserved in the interaction between light and matter is $E_{c}=P \Delta t$, where $\Delta t$ is a time interval. The magnitude of $\Delta t$ is unknown, however we know that a suitable $\Delta t$ should enable $E_{c}$ to account for the energy conserved in the photoelectric effect, in photocurrent production in semiconducting interfaces in FETs, in the activation of photosynthetic reactions, and in enabling vision in vertebrates through RGCs.

\section{Experimental Methods}

Capacitors. To test the interaction between visible and IR light and matter we used Custom Thermoelectric 07111-9L31-04B devices. These devices produce a voltage difference $\Delta V$ where there is a temperature difference $\Delta T$ across the device in agreement with the Seebeck effect $\Delta V=S \Delta T$, where $S$ is the Seebeck coefficient. However, their multilayer structure consisting of $\mathrm{AlO}-\mathrm{Cu}-\mathrm{Bi}_{2} \mathrm{Te}_{3}-$ $\mathrm{Cu}-\mathrm{AlO}$ shown in Appendix 1 can also be viewed as a capacitive structure. The basic device has a height $h=3 \mathrm{~mm}$ and a capacitance $C$ in the $\mathrm{pF}$ range. Adding insulating layers to the basic device, and varying the cross-sectional area with diameter $D$ of the illuminating laser, enable us modifying the capacitance $C$. The configurations of the devices examined in our work, alongside their $C$ and dielectric constant $\varepsilon$ values, are summarized in Appendix 1. The $\varepsilon$ values are calculated from $C=\varepsilon \varepsilon_{0} \frac{\text { area }}{h}$, where $\varepsilon_{0}=8.854 \times 10^{-12} \frac{\mathrm{F}}{\mathrm{m}}$ is the permittivity in vacuum.

Infrared sources. The characteristics of the visible and IR continuous wave (c.w.) and pulsed (PL) lasers used in our experiments are summarized in Table 1 and Table 2.

Data acquisition and modeling. Using Keithley 2000 multi-meters, we acquired voltage $\Delta V(t)$ and temperature $\Delta T(t)$ differences as a function of time $t$. The temperatures of the illuminated $\left(T_{\text {light }}(t)\right)$ and non-illuminated ( $\left.T_{n o-l i g h t}(t)\right)$ faces of the capacitors were measured using OMEGA type $\mathrm{E}$ $\mathrm{Ni}-\mathrm{Cr} / \mathrm{Cu}-\mathrm{Ni}$ thermocouple probes. We measured $\Delta V(t)$ and $\Delta T(t)$ using LabView 2012 and a National Instruments PXI-1042q communications chassis. We collected and analyzed a total of 116 data sets with different combinations of capacitor's capacitance $C$, light's average power $P$ and/or period $\tau$ for c.w. and PL lasers. Each set consists of $\Delta V(t), \Delta T(t), T_{\text {light }}(t)$ and $T_{n o-\text { light }}(t)$. We used Wolfram Mathematica 10.3 software for modeling. 
Table 1. Mode of operation, period $\tau$, maker and type, average power $P$ range, and beam diameter $D$ of the visible and IR continuous wave (c.w.) lasers used in our experiments.

\begin{tabular}{ccccc}
\hline $\begin{array}{c}\text { Mode of } \\
\text { operation }\end{array}$ & $\begin{array}{c}\text { Period } \tau \\
{[\mathrm{fs}]}\end{array}$ & Maker and type & $\begin{array}{c}\text { Average power } \\
\text { P range }[\mathrm{mW}]\end{array}$ & $\begin{array}{c}\text { Beam diameter } \\
\text { D [mm] }\end{array}$ \\
\hline c.w. & 1.77 & Viasho solid state & $25-50$ & 3 \\
c.w. & 3.55 & $\begin{array}{c}\text { ThorLabs solid state } \\
\text { IPG Photonics pumped } \\
\text { colid state }\end{array}$ & $25-1000$ & 3 \\
& 6.67 & $50-200$ & 5 \\
\hline
\end{tabular}

Table 2. Mode of operation, period $\tau$, maker and type, repetition rate $\frac{1}{T_{p}}$, pulse duration $\Delta t_{p}$, average power $P$ range, peak power $P_{\text {peak }}$ range, and beam diameter $\mathrm{D}$ of the IR pulsed (PL) lasers used in our experiments.

\begin{tabular}{cccccccc}
\hline $\begin{array}{c}\text { Mode of } \\
\text { operation }\end{array}$ & $\begin{array}{c}\text { Period } \tau \\
{[\mathrm{fs}]}\end{array}$ & $\begin{array}{c}\text { Maker and } \\
\text { type }\end{array}$ & $\begin{array}{c}\text { Repetition } \\
\text { rate } 1 / \mathrm{T}_{\mathrm{p}} \\
{[1 / \mathrm{s}]}\end{array}$ & $\begin{array}{c}\text { Pulse } \\
\text { duration } \\
\Delta \mathrm{t}_{\mathrm{p}}[\mathrm{ns}]\end{array}$ & $\begin{array}{c}\text { Average } \\
\text { power P } \\
\text { range } \\
{[\mathrm{mW}]}\end{array}$ & $\begin{array}{c}\text { Peak } \\
\text { power } \\
\mathrm{P}_{\text {peak }} \\
\text { range }\end{array}$ & $\begin{array}{c}\text { Beam } \\
\text { diameter } \\
\mathrm{D}\end{array}$ \\
\hline PL & 3.55 & $\begin{array}{c}\text { Northrop } \\
\text { Grumman } \\
\text { solid state }\end{array}$ & $4^{*} 10^{3}$ & 0.7 & $5-50$ & $\mathrm{~kW}$ & 4 \\
PL & 3.55 & $\begin{array}{c}\text { New Wave } \\
\text { Research } \\
\text { Tempest }\end{array}$ & 10 & 7 & $150-450$ & $\mathrm{MW}$ & 3 \\
\hline
\end{tabular}

\section{Results and Discussion}

To estimate the magnitude of $\Delta t$, and therefore of $E_{c}=P \Delta t$, we collected and analyzed 116 sets of voltage and temperature measurements resulting from the excitation of capacitors by lasers of average power $P$ spanning from 10 to $1 \times 10^{3}$ $\mathrm{mW}$. In our experiments, we use visible and IR lasers with wavelength $\lambda$ from 532 to $2 \times 10^{3} \mathrm{~nm}$ (i.e. from frequency $v=0.56 \mathrm{PHz}$ or period $\tau=1.77 \mathrm{fs}$, to $v=0.15 \mathrm{PHz}$ or $\tau=6.70 \mathrm{fs}$ ). We shine c.w. and PL lasers with beam diameter $D$ onto capacitors with capacitance $C$ in the $\approx 18$ to $\approx 270 \mathrm{pF}$ range (Section-2). We then monitor the voltage $\Delta V(t)$ and temperature $\Delta T(t)$ differences produced by the capacitors versus time $t$ in the 400 seconds immediately following the start of the illumination with visible or IR light. The exponential time-dependent rise of $\Delta V(t)$ occurs in the time interval we call the exponential perturbation regime (EPR). The end of the EPR marks the beginning of the steady state laser illumination (ssli) where the voltage reaches a stable value. The two regimes are labelled in Figure 1(a). From the experimental measurements we extract the amplitude of the jumps in voltage and temperature, $\Delta V$ and $\Delta T$ respectively. These jumps in voltage and temperature arise in the capacitors because the energy $E_{c}$, conserved in the interaction between visible or IR light with the capacitors, is distributed into an electrical and a thermal component. Exploiting the experimentally observed trends of $\Delta V(t)$ and $\Delta T(t)$ versus $t$, 

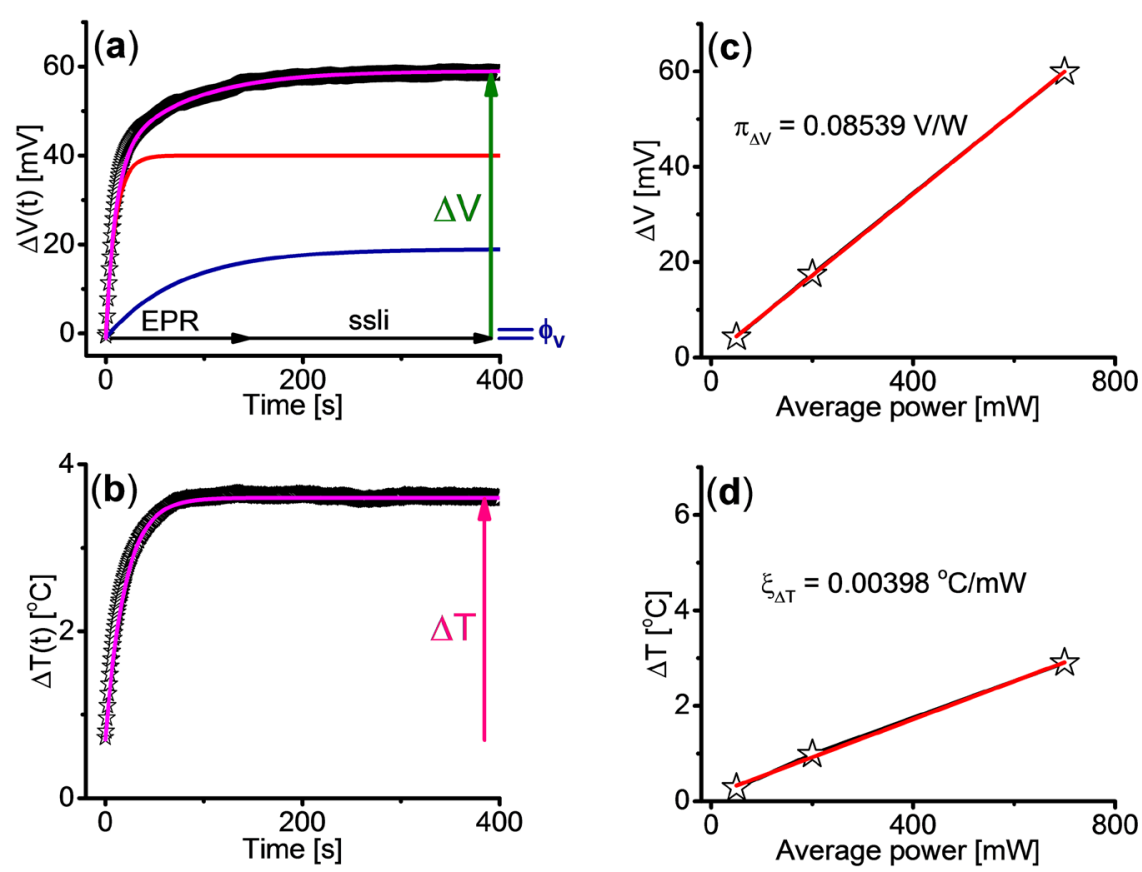

Figure 1. (a) Example of $\Delta V(t)$ trends and (b) example of $\Delta T(t)$ trends. These data were measured from a capacitor with $C=43.4 \mathrm{pF}$ upon illumination with a continuous wave (c.w.) laser with $P=700 \mathrm{~mW}, D=3 \mathrm{~mm}$, and $\tau=3.55 \mathrm{fs}$. The amplitudes of the voltage $\Delta V=60 \mathrm{mV}$ and temperature $\Delta T=2.9^{\circ} \mathrm{C}$ jumps are labeled in the panels. The exponential perturbation regime (EPR), the steady state laser illumination (ssli), and the $\phi_{V}$ offset are labeled in (a). The $\Delta V$ and $\Delta T$ jumps versus $P$ obtained on the same capacitor upon illumination with a c.w. laser with $D=3 \mathrm{~mm}$ and $\tau=3.55$ fs in the average power $P$ range from 50 to $700 \mathrm{~mW}$ are shown in panels (c) and (d). The slopes of $\Delta V$ versus $P$, and $\Delta T$ versus $P$, are the voltage responsivity $\pi_{\Delta V}$ and the temperature responsivity $\xi_{\Delta T}$, respectively.

we developed a model, based on the law of conservation of energy (derived in Appendix 2), to link the magnitude of $\Delta V$ and the evolution in time of $\Delta V(t)$ to $E_{c}, P(t), \Delta T(t)$, and $C$. Here $P(t)$ is the exponential function that describes the rise versus time of the laser's power with time constant $\tau_{P}$ such that:

$$
P(t)=-P_{0} \mathrm{e}^{-\frac{t}{\tau_{P}}}+P_{s s l i},
$$

where $P_{0} \approx P_{s s i}$, and $P_{s s l i}$ is the average power in the ssli.

The typical trends of $\Delta V(t)$ and $\Delta T(t)$ are displayed in Figure 1(a) and Figure 1(b). Similar trends with current appear in Ref. [7]. We fit the experimental data in Figure 1(a) with a sum of two exponential functions, and those in Figure 1(b) with a single exponential function. From the fitting functions we extract the amplitude of the jumps in voltage and temperature, $\Delta V$ and $\Delta T$ respectively, labelled in Figure 1(a) and Figure 1(b). On average, the time constants of $\Delta V(t)$ and $\Delta T(t)$ in the EPR are $\sim 10 \mathrm{~s}$ and $\sim 35 \mathrm{~s}$, respectively. We then plot $\Delta V$ and $\Delta T$ versus the average power $P$ of the visible or IR light, 
and observe linear trends as those illustrated in Figure 1(c) and Figure 1(d). We measure the slope of $\Delta V$ versus $P$ in Figure $1(\mathrm{c})$ to obtain the voltage responsivity $\pi_{\Delta V}$ [16], and the slope of $\Delta T$ versus $P$ in Figure $1(\mathrm{~d})$ to obtain the temperature responsivity $\xi_{\Delta T}$. The $\pi_{\Delta V}$ and $\xi_{\Delta T}$ responsivities indicate the amount of voltage and temperature produced by the capacitor at a certain average power $P$ of the visible or IR lasers.

Next, in Figure 2(a) and Figure 2(b) we plot the voltage $\pi_{\Delta V}$ and temperature $\xi_{\Delta T}$ responsivities versus the capacitance $C$ of the capacitors. We exhibit data obtained with c.w. and PL lasers. The specific PL lasers used have peak power $P_{\text {peak }}$ in the $\mathrm{kW}$ and MW ranges (Section 2). Clearly, $\pi_{\Delta V}$ increases when $C$ decreases, while, within the uncertainties, $\xi_{\Delta T}$ is not sensitive to $C$. We show additional data in Appendix 3 confirming these trends with $C$. The small uncertainties on the responsivities signify negligible defects in our devices [7]. There are no noticeable differences between the trends of the $\pi_{\Delta V}$ and $\xi_{\Delta T}$ responsivities obtained with c.w. and PL lasers. This result aligns with the findings of Geiregat et al. [17] inferred from the study of amplified spontaneous emission spectra from thin films.

In Figure 2(c) and Figure 2(d) we display the $\pi_{\Delta V}$ and $\xi_{\Delta T}$ responsivities versus the period $\tau$ of the c.w. lasers obtained using capacitors with $C=43.4$ $\mathrm{pF}$ and $C=270.5 \mathrm{pF}$. We observe an increase in $\pi_{\Delta V}$ when $\tau$ increases, i.e. toward the far IR and microwave regions of the EM spectrum. Remarkably, the uncertainties of the data from the capacitor with $C=43.4 \mathrm{pF}$ never overlap, strongly supporting that $\pi_{\Delta V}$ increases when $\tau$ increases. The temperature responsivity $\xi_{\Delta T}$ exhibits a slight increase toward the visible region of the EM spectrum. We provide additional data in Appendix 3, which confirm these trends with $\tau$.

The experimental data indicate that $\Delta V$ increases when the EM wave's average power $P$ increases. Moreover, the trends of $\pi_{\Delta V}$ versus $C$ and $\tau$ suggest that the increase in $\Delta V$ with $P$ is enhanced when $C$ decreases and $\tau$ increases. To model these trends we derive Equation (2a) and Equation (2b), which stem from the law of conservation of energy applied to the interaction between visible or IR light and a capacitor. We present the derivation of Equation (2a) and Equation (2b) in Appendix 2. A similar approach is adopted in Ref. [18]. According to our model, in each instant of time $t$ in the EPR the energy $E(t)=P(t) \Delta t$, conserved in the interaction between visible or IR light and a capacitor, is:

$$
E(t)=\frac{1}{2} C \Delta V(t)^{2}+\frac{1}{2} \frac{q(t)^{2}}{C}-\Sigma_{0} \Delta T(t)
$$

where $\Sigma_{0}$ is the entropy in a closed system, as discussed in Appendix 2. In each instant of time $t$, the variables $\Delta V(t), q(t)$, and $\Delta T(t)$ adjust their values to that of $E(t)=P(t) \Delta t$. Likewise, in the ssli the energy $E_{c}$ conserved in the interaction between light and matter is: 

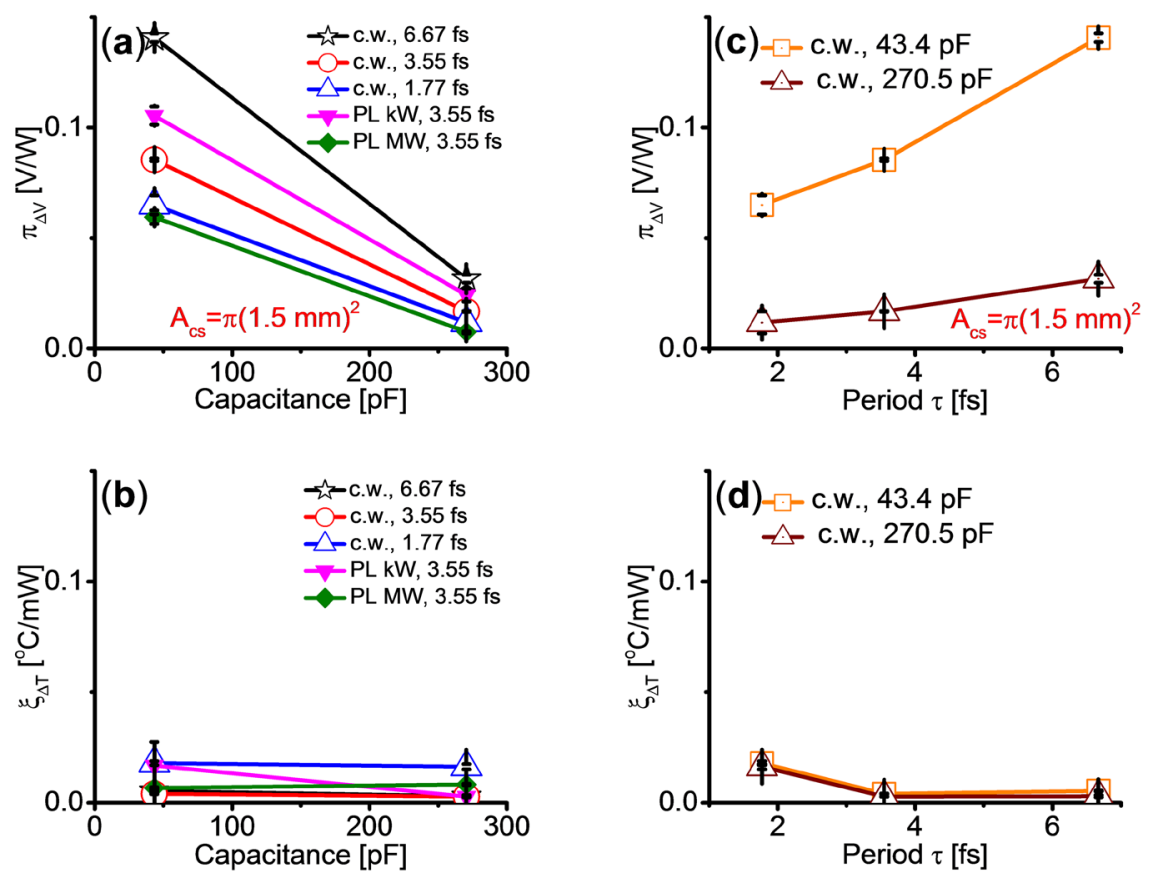

Figure 2. Trends of the (a) voltage $\pi_{\Delta V}$ and (b) temperature $\xi_{\Delta T}$ responsivities and corresponding uncertainties versus capacitance $C$ for capacitors illuminated on a cross-sectional area $A_{c s}$ with radius $\frac{D}{2}=1.5 \mathrm{~mm}$ with continuous wave (c.w.) lasers with periods $\tau=1.77 \mathrm{fs}(\lambda=532 \mathrm{~nm}), 3.55 \mathrm{fs}(\lambda=1064 \mathrm{~nm})$, and $6.67 \mathrm{fs}\left(\lambda=2 \times 10^{3} \mathrm{~nm}\right)$. We also display data obtained upon illumination of the same capacitors with pulsed (PL) lasers with $\tau=3.55$ fs and $P_{\text {peak }}$ in the $\mathrm{kW}$ and MW ranges. Trends of (c) $\pi_{\Delta V}$ and (d) $\xi_{\Delta T}$ and corresponding uncertainties versus period $\tau$ of the visible and IR lasers. The data were collected with c.w. lasers with period $\tau=1.77 \mathrm{fs}, 3.55 \mathrm{fs}$, and $6.67 \mathrm{fs}$ illuminating a cross-sectional area $A_{c s}$ with radius $\frac{D}{2}=1.5 \mathrm{~mm}$ on capacitors with $C=43.4 \mathrm{pF}$ and $C$ $=270.5 \mathrm{pF}$.

$$
E_{c}=\frac{1}{2} C \Delta V^{2}+\frac{1}{2} \frac{q_{s s l i}^{2}}{C}-\Sigma_{0} \Delta T,
$$

where $q_{s s l i}$ is the surface charge in the ssli.

From Equation (2a) we then derive the voltage difference $\Delta V(t)$ in the EPR as follows:

$$
\Delta V(t)=\sqrt{\frac{2}{C} E(t)-\frac{1}{C^{2}} q(t)^{2}+\frac{2 \Sigma_{0}}{C} \Delta T(t)} .
$$

The voltage in the EPR is $V(t)_{E P R}=\Delta V(t)+\phi_{V}$ where $\phi_{V}$ is an offset with magnitude of $\sim m V$ labelled in Figure 1(a). The offset $\phi_{V}$ is due to contributions from the environment and from the capacitor. Likewise, from Equation (2b) we obtain $\Delta V$ in the ssli as:

$$
\Delta V=\sqrt{\frac{2}{C} E_{c}-\frac{1}{C^{2}} q_{s s l i}^{2}+\frac{2 \Sigma_{0}}{C} \Delta T} .
$$

The voltage in the ssli is $V_{s s l i}=\Delta V+\phi_{V}$. Equation (3a) and Equation (3b) in- 
dicate that $\Delta V(t)$ and $\Delta V$ increase when $C$ decreases, in agreement with the experimental findings in Figure 2(a).

We now combine Equation (2) and Equation (3) to (i) determine the magnitude of $\Delta t$, (ii) unveil the value of $E_{c}$ when the experimental value of the average power $P$ is known, and (iii) clarify the dependence of $\Delta V(t), \Delta V$ and $\pi_{\Delta V}$ on the period $\tau$.

To illustrate the general method enabling us to determine the magnitude of $\Delta t$ we exploit the data in Figure 1(a) and Figure 1(b). The same method is equally effective when applied to all the 116 sets of data we measured and analyzed, whether collected with c.w. or PL lasers, in the whole average power and wavelength ranges explored, and for all capacitances considered. The method requires three steps:

Step 1: rough estimate of $E_{c}$ and $\Delta t$. The data in Figure 1(a) and Figure (b) are produced by a capacitor with $C=43.4 \mathrm{pF}$ illuminated with a c.w. laser at $\lambda=1064 \mathrm{~nm}(\tau=3.55 \mathrm{fs})$ and $P=700 \mathrm{~mW}=P_{s s l i}$. The jumps in voltage and temperature are $\Delta V=60 \mathrm{mV}$ and $\Delta T=2.9^{\circ} \mathrm{C}$. In the EPR, the time constants of $\Delta T(t)$ and $P(t)$ are $\tau_{\Delta T}=48 \mathrm{~s}$ and $\tau_{P}=1.5 \mathrm{~s}$, respectively. Knowing $C$ and $\Delta V$, from Equation (2b) we roughly estimate $E_{c}$ as $E_{c} \approx \frac{1}{2} C \Delta V^{2} \approx 78.1 \mathrm{fJ}$. Thus, $\Delta t=\frac{E_{c}}{P} \approx 111.6 \mathrm{fs}$. This small time interval is only about two orders of magnitude larger than the period $\tau=3.55 \mathrm{fs}$ actually employed in the measurement. We thus hypothesize that $\Delta t \sim \tau$ and $E_{c} \sim P \tau$. Plugging $E_{c} \sim P \tau$ in Equation (3b) for $\Delta V$ suggests that $\pi_{\Delta V}$ and $\Delta V$ increase with $\tau$ in agreement with the trends observed in Figure 2(c). Despite the agreement, the hypothesis that $\Delta t \sim \tau$ needs further support.

Step 2: modeling of $\Delta V(t)$ and search for the estimated parameters $q_{s s l i}$ and $\Sigma_{0}$. With the rough hypotheses that $\Delta t \sim \tau$ and $E(t) \sim P(t) \tau$ we model the voltage difference $\Delta V(t)$ in the EPR from Equation (3a). To reach this goal we first construct an expression for the evolution with time of the surface charge $q(t)$ (see Appendix 2). Then, we superimpose Equation (3a) to the function that fits the data in Figure 1(a). With $q_{\text {ssli }}=1.05 \mathrm{pC}$ and $\Sigma_{0}=30.5 \frac{\mathrm{fJ}}{{ }^{\circ} \mathrm{C}}$ (similar values of entropy are reported in Ref. [19]) we obtain the best fit between experimental and modeled $\Delta V(t)$ shown in Figure 3(a). The agreement is very good, despite the slight mismatch at the inflection point, which we attribute to the uncertainties in $\tau_{P}$ and $\tau_{\Delta T}$.

Step 3: refined expression for $E_{c}$ and value for $\Delta t$. By plugging into Equation (2b) for $E_{c}$ the experimental parameters and the estimated parameters found through Step 2, we extract a refined value for $E_{c} \approx 2.4 \mathrm{fJ}$ and calculate $\Delta t=\frac{E_{c}}{P}=3.4 \mathrm{fs} \approx \tau=3.55 \mathrm{fs}$ for the data in Figure 1(a). From this result we infer that $E_{c} \approx P \tau$.

The same method illustrated above leads us to the three additional examples of good match between experimental and modeled $\Delta V(t)$ shown in Figure 3(b), 

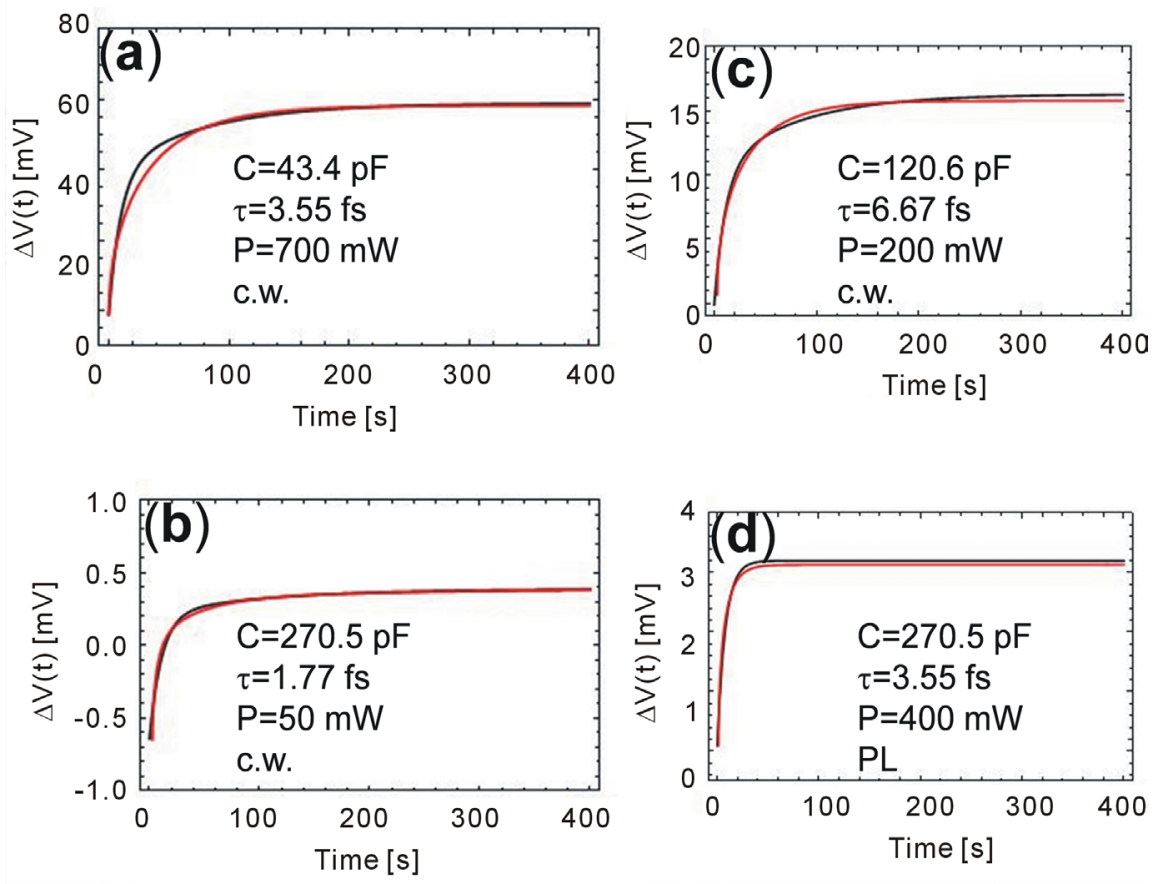

Figure 3. The function $\Delta V(t)$ obtained from Equation (3a) by hypothesizing $E(t)$ $\sim P(t) \tau$ is superimposed to the experimental data of all the 116 sets collected with either continuous wave (c.w.) or pulsed (PL) lasers and then analyzed. Here we illustrate some examples and list the experimental and estimated parameters used in the modeling from Equation (3a). (a) c.w., experimental parameters: $\Delta V=60 \mathrm{mV}, \Delta T=2.9^{\circ} \mathrm{C}, C=43.4 \mathrm{pF}$, $\tau_{\Delta T}=48 \mathrm{~s}, P=700 \mathrm{~mW}, \tau_{P}=1.5 \mathrm{~s}, \tau=3.55 \mathrm{fs}(\lambda=1064 \mathrm{~nm})$, and $\phi_{V}=-0.5 \mathrm{mV}$; estimated parameters: $q_{s s l i}=1.05 \mathrm{pC}$ and $\Sigma_{0}=30.5 \frac{\mathrm{fJ}}{{ }^{\circ} \mathrm{C}}$. In this case, $V_{s s l i}=(58.8 \pm 0.5) \mathrm{mV}$, where $V_{s s l i}$ is the voltage in the steady state laser illumination (ssli), and $\phi_{V_{\bmod }}=-6.9 \mathrm{mV}$, where $\phi_{V_{\bmod }}$ is the offset used in the modeling. (b) c.w., experimental parameters: $\Delta V=1.03 \mathrm{mV}, \Delta T=0.98^{\circ} \mathrm{C}, C=270.5 \mathrm{pF}, \tau_{\Delta T}=45 \mathrm{~s}, P=50 \mathrm{~mW}$, $\tau_{P}=7 \mathrm{~s}, \tau=1.77 \mathrm{fs}(\lambda=532 \mathrm{~nm})$, and $\phi_{V}=-0.6 \mathrm{mV}$; estimated parameters: $q_{s s l i}=0.11 \mathrm{pC}$ and $\Sigma_{0}=0.08 \frac{f J}{{ }^{\circ} \mathrm{C}} \cdot V_{s s l i}=(0.38 \pm 0.01) \mathrm{mV}$ and $\phi_{V_{\bmod }}=-0.65 \mathrm{mV}$. (c) c.w., experimental parameters: $\Delta V=16.4 \mathrm{mV}, \Delta T=1.05^{\circ} \mathrm{C}, C=120.6 \mathrm{pF}, \tau_{\Delta T}=42 \mathrm{~s}$, $P=200 \mathrm{~mW}, \tau_{P}=1 \mathrm{~s}, \tau=6.67 \mathrm{fs}\left(\lambda=2 \times 10^{3} \mathrm{~nm}\right)$, and $\phi_{V}=0.0025 \mathrm{mV}$; estimated parameters: $q_{s s l i}=0.16 \mathrm{pC}$ and $\Sigma_{0}=14.2 \frac{f J}{{ }^{\circ} \mathrm{C}} . V_{s s l i}=(16 \pm 1) \mathrm{mV}$ and $\phi_{V_{\bmod }}=0.6 \mathrm{mV} .(\mathrm{d})$ PL, experimental parameters: $\Delta V=3.1 \mathrm{mV}, \Delta T=3.7^{\circ} \mathrm{C}, C=270.5 \mathrm{pF}, \tau_{\Delta T}=18 \mathrm{~s}, P=$ $400 \mathrm{~mW}, \tau_{P}=10 \mathrm{~s}, \tau=3.55 \mathrm{fs}(\lambda=1064 \mathrm{~nm})$, and $\phi_{V}=0.17 \mathrm{mV}$; estimated parameters: $q_{s s l i}=0.23 \mathrm{pC}$ and $\Sigma_{0}=6.07 \frac{\mathrm{aJ}}{{ }^{\circ} \mathrm{C}} \cdot V_{s s l i}=(3.16 \pm 0.05) \mathrm{mV}$ and $\phi_{V_{\bmod }}=0.04 \mathrm{mV}$.

Figure 3(c) and Figure 3(d) and obtained with c.w. (Figure 3(b) and Figure 3(c)) and PL lasers (Figure 3(d)). In all cases, we calculate $\Delta t=\frac{E_{c}}{P} \approx \tau$ and derive $E_{c} \approx P \tau$. Thus, we conclude that $\Delta t=\tau$ and that the energy conserved in the interaction between visible or IR light with matter is $E_{c}=P \tau$. We thus 
rewrite Equation (3a) and Equation (3b) as:

$$
\Delta V(t)=\sqrt{\frac{2}{C} P(t) \tau-\frac{1}{C^{2}} q(t)^{2}+\frac{2 \Sigma_{0}}{C} \Delta T(t)}
$$

and

$$
\Delta V=\sqrt{\frac{2}{C} P \tau-\frac{1}{C^{2}} q_{s s l i}^{2}+\frac{2 \Sigma_{0}}{C} \Delta T} .
$$

Equation (4a) and Equation (4b) align with the experimental finding that $\pi_{\Delta V}$ increases when $\tau$ does.

In support to the equation $E_{c}=P \tau$, we point out that Har-Shemesh \& Di Piazza [20] relate power and period of a laser to the energy conserved in the interaction between high energy $\mathrm{x}$-rays and matter. Furthermore, in what follows we show how $E_{c}=P \tau$ allows us to capture the order of magnitude of the energy conserved in the photoelectric effect with $\mathrm{x}$-rays, in photocurrent production in FETs, in photosynthetic reactions, and in the generation of action potentials enabling vision in vertebrates through RGCs.

Photoelectric effect. An x-ray photon with energy $E_{x}=1.2536 \mathrm{keV}$ has a frequency $v_{p h}=0.3 \times 10^{18} \mathrm{~Hz}$ (or $\tau_{x}=3.3$ as ) derived from the equation $E_{x}=h v_{p h}$. Assuming $E_{x}=E_{c}=P_{x} \tau_{x}$ we estimate $P_{x} \approx 67 \mathrm{~W}$, which is in good agreement with the value $P_{x} \approx 100 \mathrm{~W}$ for commercial x-ray sources [21]. We note that the photon was originally defined in the conservation of energy scenario for the photoelectric effect described by $E_{c}=P_{x} \tau_{x}=n_{\tau} h v_{p h}$. In this case, the number of photons in the time interval $\tau$ is $n_{\tau}=1$ [1].

Electronic devices. Barati et al. [5] used near IR light at $\tau=3 \mathrm{fs}$ and $P=17 \mu \mathrm{W}$ to produce $E_{c}=P \tau=0.32 \mathrm{eV}$, which corresponds to the magnitude of the energy gap $E_{C B M o}-E_{C B W}=0.32 \mathrm{eV}$ between the semiconductors $\mathrm{CBs}$ at the interface in $\mathrm{MoSe}_{2} / 2 L$-WSe $\mathrm{W}_{2}$ heterostructure photocells [5]. We notice that $0.32 \mathrm{eV} \approx \frac{1}{4} h v_{p h}$, where $v_{p h}=0.3 \mathrm{PHz}$ is the frequency of the photon that corresponds to $\tau=3 \mathrm{fs}$. In this case, from $E_{c}=P \tau=n_{\tau} h v_{p h}$ we extract $n_{\tau}=\frac{1}{4}$ which means that one photon is expected to arrive on a photocell every $\approx 4 \tau$. Similarly, Sarker et al. [7] shone green light at $\tau=1.77 \mathrm{fs}$ and $P=5 \mu \mathrm{W}$ onto graphene FETs on $4 H$-SiC substrates to produce $E_{c}=P \tau=50 \mathrm{meV}$, which closely corresponds to the ionization energy (IE) of phosphorous $(60.7 \mathrm{meV})$ and nitrogen (61.4 meV) shallow donors in $4 H$-SiC [22]. The IE fixes the magnitude of the energy that electrons need to jump from the shallow donor levels to the $\mathrm{CB}$ in $4 H-\mathrm{SiC}$. From $50 \mathrm{meV}=n_{\tau} h v_{p h}$, where $v_{p h}=0.56 \mathrm{PHz}$ is the frequency of the photon with $\tau=1.77 \mathrm{fs}$, we extract $n_{\tau}=\frac{1}{47}$ which means that one photon is expected to arrive on the graphene FET every $\approx 47 \tau$.

Photosynthesis. Let us assume that the average solar light power per unit area $P \approx 136 \frac{\mathrm{mW}}{\mathrm{cm}^{2}}$ [8] [9] remains unchanged during the interaction of the light with a natural LH system. Then, in the carotenoid $S_{2}$ stimulated emission [13] with 
green light at $\tau_{\text {in }}=1.73 \mathrm{fs}$ entering a photosynthetic organism and releasing green light at $\tau_{\text {out }}=1.82 \mathrm{fs}$, the exchanged energy is $\left|P\left(\tau_{\text {out }}-\tau_{\text {in }}\right)\right| \approx 76 \mathrm{eV}$. In the absorption of green light by the BChl-a $\mathrm{Q}_{\mathrm{x}}$ state at $\tau_{\text {in }}=1.82 \mathrm{fs}$ and consequent release of yellow light at $\tau_{\text {out }}=1.93 \mathrm{fs}$, the exchanged energy is $\left|P\left(\tau_{\text {out }}-\tau_{\text {in }}\right)\right| \approx 85 \mathrm{eV}$. These values of exchanged energies enable in a small time interval the activation of multiple photosynthetic reactions requiring different activation energies per molecule (e.g. $0.5 \mathrm{eV}$ for the oxidation of cytochrome [10], or $0.72 \mathrm{eV}$ for the $\mathrm{CO}_{2}$ exchange [11], or $1.6 \mathrm{eV}$ for the $\mathrm{CO}_{2}$ oxidation [12]). The mechanism of energy transfer from light into a natural LH system and subsequent distribution into various chemical reaction pathways requiring different activation energies is called down-conversion [23]. We observe that, assuming $0.75 \mathrm{eV}$ to be the average energy per molecule required to activate one of the photosynthetic reactions [10] [11] [12] [13], we have $0.75 \mathrm{eV} \approx n_{\tau c}\left|h\left(v_{\text {out }}-v_{\text {in }}\right)\right|$ $\approx 6 \times 0.12 \mathrm{eV}$, where the photon frequencies $v_{\text {out }}$ and $v_{\text {in }}$ vary between 0.52 and $0.58 \mathrm{PHz}$ corresponding to the 1.73 to $1.93 \mathrm{fs} \tau$ range. The equation $n_{\tau c}=6$ indicates that about six couples of photons are required in the time interval $\tau$ to activate one photosynthetic reaction.

Vision in vertebrates. Let us assume that solar green light at $\tau=1.82 \mathrm{fs}$ $\left(v_{p h}=0.55 \mathrm{PHz}\right)$ and $P \approx 136 \frac{\mathrm{mW}}{\mathrm{cm}^{2}}$ [8] [9] illuminates the RGCs altering their capacitance by $\approx 0.2 \mathrm{pF}$ [14]. From $E_{c}=P \tau \approx \Pi=\frac{1}{2} C V_{a p}^{2}$ we obtain an action potential $V_{a p} \approx 50 \mathrm{mV}$ in excellent agreement with the experimental value of $\approx 70 \mathrm{mV}$ [14]. The term $\Pi$ is the energy that arrives on the capacitors after the chemical phenomena occurring in the visual cascade triggered by the absorption of light in the retina [24]. From $E_{c}=P \tau=1.55 \mathrm{keV}=n_{\tau} h v_{p h}$, we estimate $n_{\tau} \approx 680$ which means that about 680 photons are expected to reach a RGC in a time interval $\approx \tau$. We find noteworthy the similarity between the magnitudes of $C$ and $V_{a p}$ produced by the solar visible light in the RGCs, and the magnitudes of $C$ and $\Delta V$ in Figure 1(a) produced by near IR light.

We explore now other consequences of $E_{c}=P \tau$ being the energy conserved in the interaction between matter and light in the low frequency range of the EM spectrum:

Microwave technology. Electromagnetic waves can generate, or be generated by, mechanical motion [25] such as vibrations [26], rotations [27] [28] [29] at the nuclear [29], molecular [25] [26], and astrophysical [27] [28] levels. Thus, we anticipate that $E_{c}=P \tau$ could affect the mechanical design of ordinary tools that exploit the interaction between microwaves and matter. As an example, we illustrate the case of a microwave oven, which operates at $P_{\mu} \approx 1500 \mathrm{~W}$ and $v_{\mu}=2.45 \mathrm{GHz}$ (or $\tau_{\mu}=0.41 \mathrm{~ns}$ ) [30]. Let us imagine the situation in which, inside the oven, a support plate rotates with a cup on top of it at a rate $\rho_{\text {rot }}$ and period $\mathrm{T}_{r o t}$. Assuming a $1 \mathrm{~kg}$ support plate with a $16 \times 10^{-2} \mathrm{~m}$ radius (notice that $\lambda_{\mu}=12.4 \times 10^{-2} \mathrm{~m}$ ) topped by a $0.8 \mathrm{~kg}$ cup full of water with a $5 \times 10^{-2} \mathrm{~m}$ radius, we calculate a moment of inertia $I_{\exp } \approx 138 \times 10^{-4} \mathrm{~kg} \cdot \mathrm{m}^{2}$ for the support plate + 
cup system. Since microwaves can transfer mechanical/rotational energy to the support plate + cup system, we depict the process as $E_{c}=P_{\mu} \tau_{\mu} \approx \frac{1}{2} I_{\exp } \rho_{r o t}^{2}$, and extract $\rho_{r o t} \approx 0.01 \frac{1}{\mathrm{~s}}$ and $\mathrm{T}_{r o t} \approx 100 \mathrm{~s}$. Technological reasons might select $\mathrm{T}_{r o t} \approx 12 \mathrm{~s}$, the value actually measured. Nevertheless, the estimated and measured values of $\mathrm{T}_{\text {rot }}$ are in good agreement.

Radio wave energy harvesting. We collected on a capacitor with capacitance $C=37 \mathrm{pF}$ the radio waves emitted and received by a cellular phone tuned to the Global System for Mobile communications (GSM) frequency of $v_{G S M}=900 \mathrm{MHz}$ $\left(\tau_{G S M}=1.1 \mathrm{~ns}\right)$. We observed the production of a $\Delta V_{G S M}=0.095 \mathrm{mV}$ with a time constant $\tau_{\Delta V_{G S M}}=95 \mathrm{~s}$ and $\Delta T_{G S M}=0.02^{\circ} \mathrm{C}$. Using the approximate expression $E_{c}=P_{G S M} \tau_{G S M} \approx \frac{1}{2} C \Delta V_{G S M}^{2}$ derived from Equation (2b) we estimate $P_{G S M}=0.15 \mathrm{nW}, E_{c}=P_{G S M} \tau_{G S M}=0.165 \mathrm{aJ}$, and expect a voltage responsivity $\frac{\Delta V_{G S M}}{P_{G S M}}=\pi_{\Delta V} \approx 0.6 \times 10^{6} \frac{\mathrm{V}}{\mathrm{W}}$, about six orders of magnitude larger than that obtained with near IR light in Figure 2(c). For comparison, the best pyroelectric detectors exhibit a $\pi_{\Delta V} \approx 10^{4} \frac{\mathrm{V}}{\mathrm{W}}$ [16]. This finding pushes forward the plan to harvest, as an alternative source of renewable and sustainable energy, the radio waves dispersed in the environment after being used in telecommunications. This effort requires a systematic exploration of the behavior of temperature, entropy and time constants in the process of energy transfer, an effort that soon we plan on undertaking.

\section{Summary and Conclusions}

By revisiting the wave nature of light, we investigate the magnitude of the energy $E_{c}$ conserved in light's interaction with matter. Upon combing experimental measurements and simulations based on the law of conservation of energy, we find that $E_{c}=P \tau=n_{\tau} h v_{p h}$, where $P$ and $\tau$ are the power and the period of the electromagnetic (EM) wave, $n_{\tau}$ is the number of photons in a time interval equal to $\tau, h$ is Planck's constant, and $v_{p h}$ is the photon's frequency. We highlight that the magnitude of the energy conserved $E_{c}$ is defined in a time interval equal to $\tau$ both for light as wave, for which $E_{c}=P \tau$, and for light as particle, for which $E_{c}=n_{\tau} h v_{p h}$. By addressing the interaction of light with matter in the photoelectric effect, in photo-excited field effect transistors, in photosynthesis and in vertebrate's vision, we show that $E_{c}=P \tau=n_{\tau} h v_{p h}$ is valid independently of the employed photo-detecting device and in the whole EM spectrum.

In addition, as a consequence of the law of conservation, we notice that the energy $E_{c}$ constraints the magnitude of the variables involved in the energy exchange between light and a specific photo-detecting device, and imposes a reciprocal self-limitation among those variables. In our experiment, for example, 
the magnitude of $E_{c}$, in cooperation with capacitor's capacitance $C$ and light's period $\tau$, dictates the magnitude of the voltages produced by the capacitors and provides a strategy to estimate the magnitude of entropy. In photo-excited field effect transistors, $E_{c}$ constraints the magnitude of the energy supplied to electrons thus promoting or suppressing photocurrent production. Similarly, the magnitude of the capacitance $C$ in retinal ganglion cells evolved in time in harmony with the power $P$ and period $\tau$ of the solar light such that $E_{c}$ can elicit action potentials with the proper value to enable vision in vertebrates.

Finally, the finding that the voltage responsivity of capacitors increases with $\tau$ and is about six orders of magnitude larger with radio waves than with visible and infrared light has strong implications in the search for alternative sources of renewable and sustainable energy: it suggests that it would be very efficient to harvest the radio waves dispersed in the environment after being used in telecommunications. This effort requires a systematic exploration of the behavior of temperature, entropy and time constants in the process of energy transfer, an effort that soon we plan on undertaking.

In conclusion, our results suggest that the wave-nature of light is necessary and sufficient to describe the energy conserved in light-matter interaction. The number of photons involved can be determined as a simple consequence, and the magnitude of the phenomena arising from light-matter interaction and their connection can be established once the wave properties of light are known.

\section{Acknowledgements}

This work was supported by the U.S. Office of Naval Research (ONR) awards \# N000141410378 and N000141512158, the JMU 4-VA Consortium (2016-2017), the JMU-Madison Trust-Fostering Innovation and Strategic Philanthropy-Innovation Grant-2015, the Thomas F. Jeffress and Kate Miller Jeffress Memorial Trust, Grant \# J-1053, the JMU Center for Materials Science, the JMU Department of Physics and Astronomy, and the JMU College of Science and Mathematics. A portion of this research was conducted at the Center for Nanophase Materials Sciences, which is a Department of Energy (DOE), Office of Science User Facility at the Oak Ridge National Laboratory under the Grant CNSM2014-R16. The authors thank M. Currie and V. D. Wheeler (Naval Research Laboratory-NRL) for their help in the experiments, and B. C. Utter (Bucknell University), J. C. Zimmerman (JMU), W. W. Shiflet (JMU), G. N. Parsons (NCSU), and F. Segatta (University of Bologna, Italy) for fruitful discussions.

\section{References}

[1] Wheaton, B.R. (2009) Photoelectric Effect. In: Greenberger, D., Hentschel, K. and Weinert, F., Eds., Compendium of Quantum Physics, Springer, Berlin, Heidelberg, 472-475. https://doi.org/10.1007/978-3-540-70626-7_143

[2] Kovac, J., Scarel, G., Sakho, O. and Sancrotti, M. (1995) An Experimental Study of the Electronic Structure of $\mathrm{C}_{60}$ Films Grown at the Ag (110) Surface. Journal of Electron Spectroscopy and Related Phenomena, 72, 71-75. 
https://doi.org/10.1007/978-3-540-70626-7_143

[3] Barr, T.L. (1994) Modern ESCA: The Principles and Practice of X-Ray Photoelectron Spectroscopy. Taylor and Francis, Abingdon-on-Thames.

[4] Lee, H.L. and Flynn, N.T. (2006) X-Ray Photoelectron Spectroscopy. In: Vij, D., Ed., Handbook of Applied Solid State Spectroscopy, Springer, Boston, 485-507. https://doi.org/10.1007/0-387-37590-2_11

[5] Barati, F., Grossnickle, M., Su, S., Lake, R.L., Aji, V. and Gabor, N.M. (2017) Hot Carrier-Enhanced Interlayer Electron-Hole Pair Multiplication in 2D Semiconductor Heterostructure Photocells. Nature Nanotechnology, 12, 1134-1139. https://doi.org/10.1038/nnano.2017.203

[6] Adinolfi, V. and Sargent, E.H. (2017) Photovoltage Field-Effect Transistors. Nature, 542, 324-327. https://doi.org/10.1038/nature21050

[7] Sarker, B.K., Cazalas, E., Chung, T.-F., Childres, I., Jovanovic, I. and Chen, Y.P. (2017) Position-Dependent and Millimeter-Range Photodetection in Phototransistors with Micrometer-Scale Graphene on SiC. Nature Nanotechnology, 12, 668-674. https://doi.org/10.1038/nnano.2017.46

[8] University of Colorado at Boulder. The Average Solar Irradiance Is $136.05 \frac{\mathrm{mW}}{\mathrm{cm}^{2}}$ According to Data Provided by the Laboratory for Atmospheric and Space Physics.

[9] Soum-Glaude, A., Le Gal, A., Bichotte, M., Escape, C. and Dubost, L. (2017) Optical Characterization of $\mathrm{TiAlN}_{\mathrm{x}} / \mathrm{TiAlN}_{\mathrm{y}} / \mathrm{Al}_{2} \mathrm{O}_{3}$ Tandem Solar Selective Absorber Coatings. Solar Energy Materials \& Solar Cells, 170, 254-262. https://doi.org/10.1016/j.solmat.2017.06.007

[10] de Vault, D. and Chance, B. (1966) Studies of Photosynthesis Using a Pulsed Laser. Biophysical Journal, 6, 825-847. https://doi.org/10.1016/S0006-3495(66)86698-5

[11] Gifford, R.M. and Musgrave, R.B. (1972) Activation Energy and Limiting Factors in Photosynthesis. Australian Journal of Biological Sciences, 25, 419-423.

https://doi.org/10.1071/BI9720419

[12] Mayer, S.W. (1969) Estimation of Activation Energies for Nitrous Oxide, Carbon Dioxide, Nitrogen Dioxide, Nitric Oxide, Oxygen and Nitrogen Reactions by a Bond-Energy Method. The Journal of Physical Chemistry, 73, 3941-3946. https://doi.org/10.1021/j100845a064

[13] Segatta, F., Cupellini, L., Jurinovich, S., Mukamle, S., Dapor, M., Taioli, S., Garavelli, M. and Mennucci, B. (2017) A Quantum Chemical Interpretation of Two-Dimensional Electronic Spectroscopy of Light-Harvesting Complexes. Journal of the American Chemical Society, 139, 7558-7567. https://doi.org/10.1021/jacs.7b02130

[14] Kim, M.-H., Vickers, E. and von Gersdorff, H. (2012) Patch-Clamp Capacitance Measurements and $\mathrm{Ca}^{2+}$ Imaging at Single Nerve Terminals in Retinal Slices. Journal of Visualized Experiments, 59, e3345.

[15] Poynting, J.H. (1884) On the Transfer of Energy in the Electromagnetic Field. Philosophical Transactions of the Royal Society of London, 175, 343-361. https://doi.org/10.1098/rstl.1884.0016

[16] Zhao, D., Fabiano, S., Berggren, M. and Crispin, X. (2017) Ionic Thermoelectric Gating Organic Transistors. Nature Communications, 8, 14214.

https://doi.org/10.1098/rstl.1884.0016

[17] Geiregat, P., Houtepen, A.J., Sagar, L.K., Infante, I., Zapata, F., Grigel, V., Allan, G., Delerue, C., Van Thourhout, D. and Hens, Z. (2018) Continuous-Wave Infrared Optical Gain and Amplified Spontaneous Emission at Ultralow Threshold by Col- 
loidal HgTe Quantum Dots. Nature Materials, 17, 35-40. https://doi.org/10.1038/nmat5000

[18] Bagci, T., Simonsen, A., Schmid, S., Villanueva, L.G., Zeuthen, E., Appel, J., Taylor, J.M., Sørensen, A., Usami, K., Schliesser, A. and Polzik, E.S. (2014) Optical Detection of Radio Waves through a Nanomechanical Transducer. Nature, 507, 81-85. https://doi.org/10.1038/nature13029

[19] Wu, Z., Płucienik, A., Feiten, F.E., Naschitzki, M., Wachsmann, W., Gewinner, S., Schöllkopf, W., Staemmler, V., Kuhlenbeck, H. and Freund, H.-J. (2017) Vibrational Action Spectroscopy of Solids: New Surface-Sensitive Technique. Physical Review Letters, 119, Article ID: 136101. https://doi.org/10.1103/PhysRevLett.119.136101

[20] Har-Shemesh, O. and Di Piazza, A. (2012) Peak intensity Measurement of Relativistic Lasers via Nonlinear Thomson Scattering. Optics Letters, 37, 1352-1354. https://doi.org/10.1364/OL.37.001352

[21] Information from ScientaOmicron Inc. and Physical Electronics Inc.

[22] Ivanov, I.G., Henry, A. and Janzén, E. (2005) Ionization Energies of Phosphorus and Nitrogen Donors and Aluminum Acceptors in $4 \mathrm{H}$ Silicon Carbide from the Donor-Acceptor Pair Emission. Physical Review B, 71, Article ID: 241201(R). https://doi.org/10.1103/PhysRevB.71.241201

[23] Dean, J.C., Mirkovic, T., Toa, Z.S.D., Oblinsky, D.G. and Scholes, G.D. (2016) Vibronic Enhancement of Algae Light Harvesting. Chemistry, 1, 858-872. https://doi.org/10.1016/j.chempr.2016.11.002

[24] Nelson, P.C. (2017) From Photon to Neuron: Light, Imaging, Vision. Princeton University Press, Princeton.

[25] Garcia-López, V., Chen, F., Nilawski, L.G., Duret, G., Aliyan, A., Kolomeisky, A.B., Robinson, J.T., Wang, G., Pal, R. and Tour, J.M. (2017) Molecular Machines Open Cell Membranes. Nature, 548, 567-572. https://doi.org/10.1038/nature23657

[26] Stensitzki, T., Yang, Y., Kozich, V., Ahmed, A.A., Kössl, F., Kühn, O. and Heyne, K. (2018) Acceleration of a Ground-State Reaction by Selective Femtosecond-Infrared-Laser-Pulse Excitation. Nature Chemistry, 10, 126-131. https://doi.org/10.1038/nchem.2909

[27] Xu, Y., Cao, W., Ahn, K., Jing, J., Chae, J., Huang, N., Deng, N., Gary, D.E. and Wang, H. (2018) Transient Rotation of Photospheric Vector Magnetic Fields Associated with a Solar Flare. Nature Communications, 9, Article No. 46.

[28] Smit, R., Bouwens, R.J., Carniani, S., Oesch, P.A., Labbé, I., Illingworth, G.D., van der Werf, P., Bradley, L.D., Gonzalez, V., Hodge, J.A., Holwerda, B.W., Maiolino, R. and Zheng, W. (2018) Rotation in [C II]-Emitting Gas in Two Galaxies at a Redshift of 6.8. Nature, 553, 178-181. https://doi.org/10.1038/nature24631

[29] Yan, W., Fruhling, C., Golovin, G., Heden, D., Luo, J., Zhang, P., Zhao, B., Zhang, J., Liu, C., Chen, M., Chen, S., Banerjee, S. and Umstadter, D. (2017) High-Order Multiphoton Thomson Scattering. Nature Photonics, 11, 514-520.

[30] Vollmer, M. (2004) Physics of the Microwave Oven. Physics Education, 39, 74-81. https://doi.org/10.1088/0031-9120/39/1/006

[31] Chandler, D. (1987) Introduction to Modern Statistical Mechanics. Oxford University Press Inc., Oxford.

[32] Gordon, A.L., Schwab, Y., Lang, B.N., Gearhart, G.P., Jobin, T.R., Kaczmar, J.M., Marinelli, Z.J., Mann, H.S., Utter, B.C. and Scarel, G. (2015) Decoupling the Electrical and Entropic Contributions to Energy Transfer from Infrared Radiation to a Power Generator. World Journal of Condensed Matter Physics, 5, 301-318. 
https://doi.org/10.4236/wjcmp.2015.54031

[33] Afanas'ev, V.V. (2012) Internal Photoemission Spectroscopy: Principles and Applications. Elsevier Science, New York.

[34] Baker-Jarvis, J. and Kim, S. (2012) The Interaction of Radio-Frequency Fields with Dielectric Materials at Macroscopic to Mesoscopic Scales. Journal of Research of NIST, 117, 1-60.

[35] Ciappina, F., Pérez-Hernández, J.A., Landsman, A.S., Okell, W.A., Zherebtsov, S., Förg, B., Schötz, J., Seiffert, L., Fennel, T., Shaaran, T., Zimmermann, T., Chacón, A., Guichard, R., Zaïr, A., Tisch, J.W.G., Marangos, J.P., Witting, T., Braun, A., Maier, S.A., Roso, L., Krüger, M., Hommelhoff, P., Kling, M.F., Krausz, F. and Leenstein, M. (2017) Attosecond Physics at the Nanoscale. Reports on Progress in Physics, 80, Article ID: 054401. https://doi.org/10.1088/1361-6633/aa574e

[36] Jones, R.C. (1941) A New Calculus for the Treatment of Optical Systems. I. Description and Discussion of the Calculus. Journal of the Optical Society of America, 31, 488-493. https://doi.org/10.1364/JOSA.31.000488

[37] Jones, R.C. (1941) A New Calculus for the Treatment of Optical Systems. III. The Sohncke Theory of Optical Activity. Journal of the Optical Society of America, 31, 500-503. https://doi.org/10.1364/JOSA.31.000500

[38] Jones, R.C. (1942) A New Calculus for the Treatment of Optical Systems. IV. Journal of the Optical Society of America, 32, 486-493. https://doi.org/10.1364/JOSA.32.000486

[39] St. John, T.C., Marinelli, Z.J., Kaczmar, J.M., Given, R.P., Wenger, K.S., Utter, B.C. and Scarel, G. (2016) Conversion of Infrared Light into Usable Energy. Proceedins of SPIE, 9927, 99270C. 


\section{Appendix1. Schematics of the Capacitors and Summary Their Characteristics}

In this section we illustrate the structure of the Custom Thermoelectric 07111-9L31-04B devices used in our experiments. Figure A1 shows the schematics of a single capacitor and that of a single capacitor with additional insulating tape (IT) on the illuminated face. The IT consists of heavy cotton cloth pressure sensitive tape with strong adhesive and tensile properties. We use the IT to modify the capacitance $C$ of the capacitors. We highlight the multy-layer structure consisting of $\mathrm{AlO}-\mathrm{Cu}-\mathrm{Bi}_{2} \mathrm{Te}_{3}-\mathrm{Cu}-\mathrm{AlO}$. The capacitor has a thickness $h$ $=3 \mathrm{~mm}$. The cross-sectional area $A_{c s}$ of the visible or IR laser has diameter $D$ and corresponds to the illuminated area of the capacitor. Figure A2 depicts the schematics of two capacitors in series, one with and one without the additional IT. Placing two capacitors in series is an alternative way to modify the capacitance $C$. The characteristics of the capacitors, their $C$ and dielectric constant $\mathcal{E}$ values, are summarized in Tables $\mathrm{A} 1$ and Table $\mathrm{A} 2$ for the single and the two capacitors respectively. The $\varepsilon$ values are calculated from $C=\varepsilon \varepsilon_{0} \frac{\text { area }}{h}$, where $\varepsilon_{0}=8.854 \times 10^{-12} \frac{\mathrm{F}}{\mathrm{m}}$ is the permittivity in vacuum.
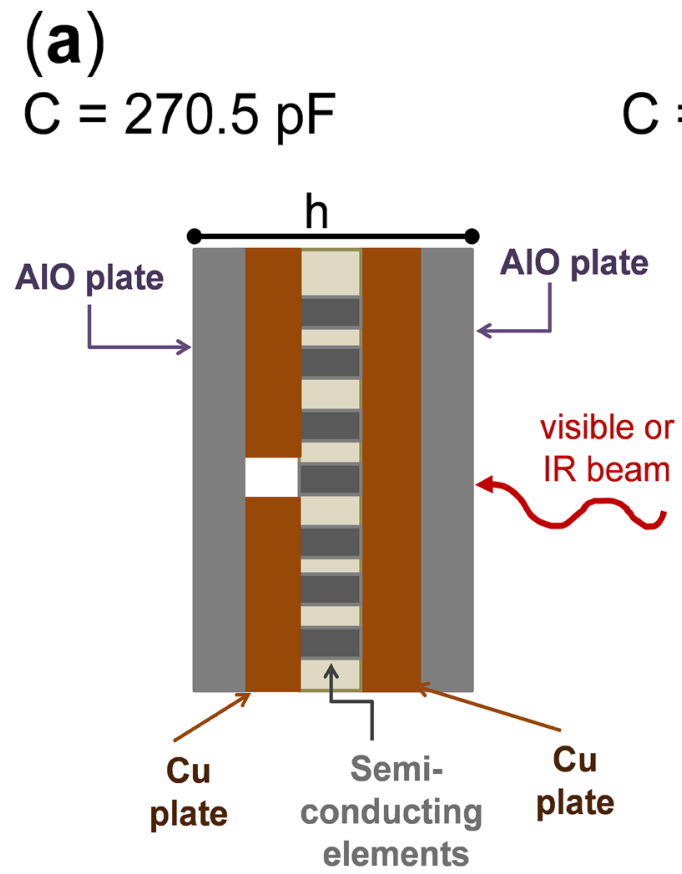

$\left(\mathrm{Bi}_{2} \mathrm{Te}_{3}\right)$
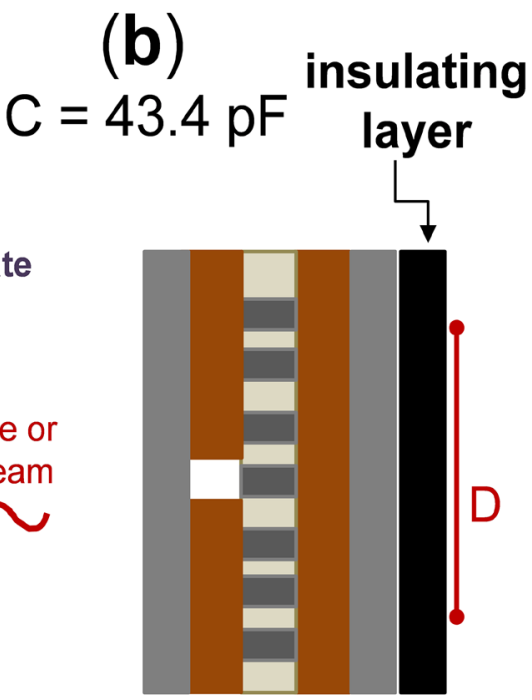

Figure A1. Schematics of (a) a single capacitor, and (b) a single capacitor with additional insulating tape (IT). The IT consists of heavy cotton cloth pressure sensitive tape with strong adhesive and tensile properties. We use the IT to modify the capacitance $C$ of the capacitor. The multy-layer structure consisting of $\mathrm{AlO}-\mathrm{Cu}-\mathrm{Bi}_{2} \mathrm{Te}_{3}-\mathrm{Cu}-\mathrm{AlO}$ is illustrated. The capacitor has a thickness $h=3 \mathrm{~mm}$. The cross-sectional area $A_{c s}$ of the visible or IR laser has diameter $\mathrm{D}$ and corresponds to the illuminated area of the capacitor. 


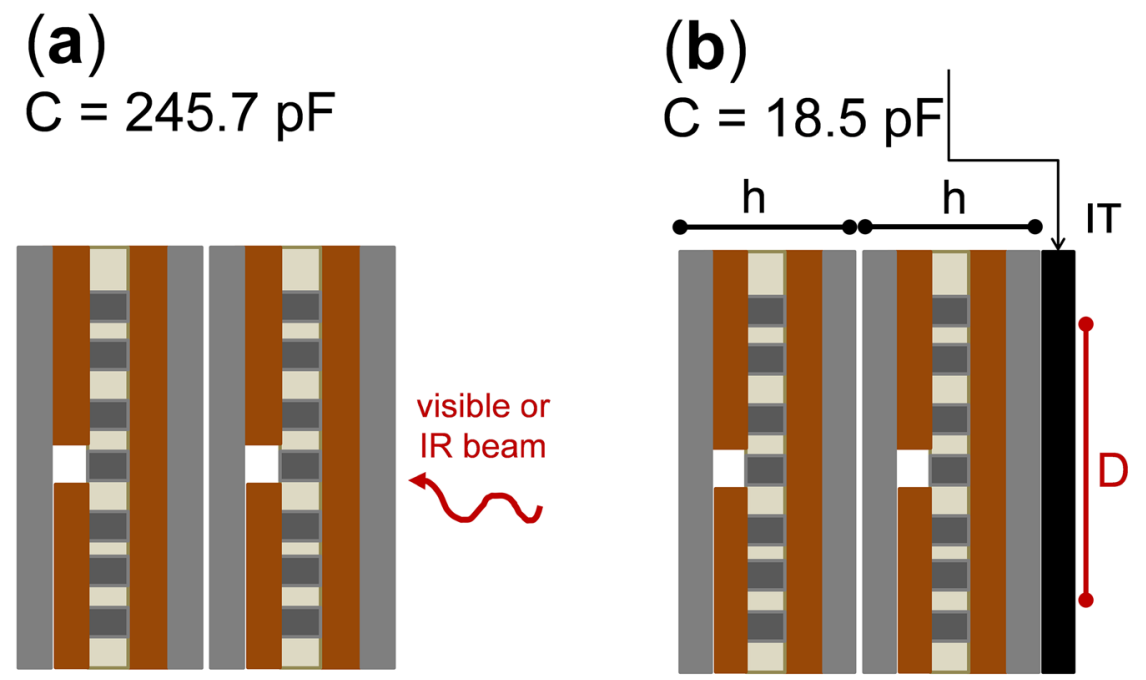

Figure A2. Schematics of (a) the two capacitors, and (b) two capacitors with additional insulating tape (IT). The other symbols are defined as in Figure A1.

Table A1. Layer structure, diameter $D$ of the illuminated area, capacitance $C$, and dielectric constant $\varepsilon$ of the single capacitors used for the data presented in the main text. We modify the $C$ values using insulating tape (IT), a pressure-sensitive tape made of heavy cotton cloth with strong adhesive and tensile properties.

\begin{tabular}{cccc}
\hline Device description & $\begin{array}{c}\text { Beam diameter } \\
\text { D [mm }]\end{array}$ & $\begin{array}{c}\text { Capacitance C } \\
{[\mathrm{pF}]}\end{array}$ & $\begin{array}{c}\text { Dielectric constant } \\
\boldsymbol{1}\end{array}$ \\
\hline 1 capacitor & 3 & 270.5 & 17312 \\
1 capacitor & 4 & 481.0 & 17316 \\
1 capacitor & 5 & 751.5 & 17315 \\
1 capacitor + IT & 3 & 43.4 & 2995.4 \\
1 capacitor + IT & 4 & 77.3 & 3001.0 \\
1 capacitor + IT & 5 & 120.6 & 2996.4 \\
\hline
\end{tabular}

Table A2. Layer structure, diameter $D$ of the illuminated area, capacitance $C$, and dielectric constant $\varepsilon$ of the two capacitors used to produce the results presented in the Figure A7. Also for two capacitors, we modify the $C$ values using insulating tape (IT), a pressure-sensitive tape made of heavy cotton cloth with strong adhesive and tensile properties.

\begin{tabular}{cccc}
\hline Device description & $\begin{array}{c}\text { Beam diameter D } \\
{[\mathrm{mm}]}\end{array}$ & $\begin{array}{c}\text { Capacitance C } \\
{[\mathrm{pF}]}\end{array}$ & $\begin{array}{c}\text { Dielectric constant } \\
\boldsymbol{\varepsilon}\end{array}$ \\
\hline 2 capacitors & 3 & 245.7 & 30832.8 \\
2 capacitors & 4 & 436.7 & 30825.8 \\
2 capacitors & 5 & 682.4 & 30828.3 \\
2 capacitors + IT & 3 & 18.5 & 2785.9 \\
2 capacitors + IT & 4 & 32.95 & 2791.0 \\
2 capacitors + IT & 5 & 51.4 & 2791.9 \\
\hline
\end{tabular}




\section{Appendix 2. Model}

\section{A2-A. Conservation of energy}

To justify the observed magnitudes and trends of the voltage responsivity $\pi_{\Delta V}$ of capacitors with capacitance $C$ illuminated by visible and infrared (IR) lasers with average power $P$ and period $\tau$, we exploit the law of conservation of energy [18]. With $P$ in the 10 to $1 \times 10^{3} \mathrm{~mW}$ range, light interacts with matter by transferring energy which subsequently is distributed into an electrical and a thermal component. Thus, we describe as follows the total differential $\mathrm{d} E$ of the energy transferred from the visible and IR lasers to the capacitor on an area $A_{c s}$ with diameter $D$ corresponding to the cross-section of the laser beam:

$$
\mathrm{d} E=q \mathrm{~d} \Delta V+\Delta V \mathrm{~d} q-\Sigma \mathrm{d} \Delta T-\Delta T \mathrm{~d} \Sigma,
$$

where $q=\sigma A_{c s}$ is charge, $\sigma=\frac{q}{A_{c s}}$ is surface charge density, and $\Sigma$ is entropy. The thermal component of Equation (A1) is preceeded by a negative sign to signify that part of the energy transferred to the capacitor as electrical energy is thermally dissipated. Initially we hypothesize that capacitance $C$ is such that $C=\frac{q}{\Delta V}$ : we will refine this assumption in Sub-section A2-D. Hypothesizing that $C=\frac{q}{\Delta V}$ enables us to separate the terms containing $q$ and $\Delta V$, thus reducing Equation (A1) to:

$$
\mathrm{d} E=C \Delta V \mathrm{~d} \Delta V+\frac{q}{C} \mathrm{~d} q-\Sigma \mathrm{d} \Delta T,
$$

where we assume $\mathrm{d} \Sigma$ to be negligible. In a generalized grand-canonical ensemble [31], the entropy $\Sigma$ is the Legendre transformation of $\Sigma_{0}$, the entropy of the canonical ensemble, such that $\Sigma=\Sigma_{0}-k_{B} \beta q \Delta V \quad$ [31], where $k_{B}=1.38 \times 10^{-23} \frac{\mathrm{J}}{\mathrm{K}}$ is Boltzmann's constant, and $\beta=\frac{1}{k_{B} T}$. Therefore:

$$
\mathrm{d} E=C \Delta V \mathrm{~d} \Delta V+\frac{q}{C} \mathrm{~d} q-\Sigma_{0} \mathrm{~d} \Delta T+k_{B} \beta q \Delta V \mathrm{~d} \Delta T .
$$

Assuming $k_{B} \beta q \Delta V$ to give just a slight correction to $\Sigma_{0}, \mathrm{~d} E$ is further reduced to:

$$
\mathrm{d} E \approx C \Delta V \mathrm{~d} \Delta V+\frac{q}{C} \mathrm{~d} q-\Sigma_{0} \mathrm{~d} \Delta T .
$$

In the exponential perturbation regime (EPR), at the start of the illumination of the capacitor with visible or IR light, the energy conserved in each instant of time $t$ is $E(t)=P(t) \Delta t$. Here $P(t)$ is the exponential function that describes the rise in time of the laser's power with a time constant $\tau_{P}$ such that:

$$
P(t)=-P_{0} \mathrm{e}^{-\frac{t}{\tau_{P}}}+P_{s s l i},
$$

where $P_{0} \approx P_{\text {ssli }} \approx P$ and $P$ is the average power in the steady state laser illumination (ssli) regime [32]. In the ssli the energy conserved is $E_{c}=P \Delta t$. To obtain 
$E(t)$ and $E_{c}$, we integrate the variables $\Delta V, E, q$, and $\Delta T$ between their minima and maxima values:

$$
\int_{\Delta E_{\min }}^{\Delta E_{M A X}} \mathrm{~d} E=C \int_{\Delta V_{\min }}^{\Delta V_{M A X}} \Delta V \mathrm{~d} \Delta V+\frac{1}{C} \int_{q_{\min }}^{q_{M A X}} q \mathrm{~d} q-\Sigma_{0} \int_{\Delta T_{\min }}^{\Delta T_{M A X}} \mathrm{~d} \Delta T .
$$

We redefine the variables such that their minima are set at zero in their respective units and their maxima correspond to the values of the variables at an arbitrary instant of time $t$ such that $\Delta V_{\max }=\Delta V(t), \Delta E_{\max }=\Delta E(t)$, $q_{\max }=q(t)$, and $\Delta T_{\max }=\Delta T(t)$. In the EPR the integration leds to:

$$
E(t)=P(t) \Delta t=\frac{1}{2} C \Delta V(t)^{2}+\frac{1}{2} \frac{q(t)^{2}}{C}-\Sigma_{0} \Delta T(t) .
$$

In the ssli the integration gives:

$$
E_{c}=P \Delta t=\frac{1}{2} C \Delta V^{2}+\frac{1}{2} \frac{q_{s s l i}^{2}}{C}-\Sigma_{0} \Delta T,
$$

where $q_{s s l i}$ is the surface charge in the ssli on the area $A_{c s}$.

To model in the EPR the measured voltage difference $\Delta V(t)$, we rearrange Equation (A7a) such that:

$$
\Delta V(t)=\sqrt{\frac{2}{C} P(t) \Delta t-\frac{1}{C^{2}} q(t)^{2}+\frac{2 \Sigma_{0}}{C} \Delta T(t)}
$$

The voltage in the EPR is $V(t)_{E P R}=\Delta V(t)+\phi_{V}$ where $\phi_{V}$ is an offset with magnitude of $\sim m V$. The offset $\phi_{V}$ is due to contributions from the environment and from the capacitor. In the ssli instead, $\Delta V$ is:

$$
\Delta V=\sqrt{\frac{2}{C} P \Delta t-\frac{1}{C^{2}} q_{s s l i}^{2}+\frac{2 \Sigma_{0}}{C} \Delta T} .
$$

The voltage in the ssli is $V_{s s l i}=\Delta V+\phi_{V}$. In the main text we use Equation (A8a) and Equation (A8b) to model the experimental data obtained with both continuous wave (c.w.) and pulsed (PL) lasers.

\section{A2-B. Model for the charge density variation in time, $\sigma(t)$.}

Of all the terms in Equation (A8a), $\frac{q(t)}{C}$ is the least constrained by experimental parameters. Thus, we exploit the surface charge $q(t)$ to achieve the best fit between modeled and experimental data. To do so, we carefully develop a model for $q(t)$, which is related to the evolution with time in the EPR of the surface charge density, $\sigma(t)$ [33], perturbed by the visible and IR lasers illuminating the capacitors. The rate of energy transferred from the visible and IR lasers to the area $A_{c s}$ with diameter $D$ is the Poynting vector $|\boldsymbol{S}|=|\boldsymbol{E} \times \boldsymbol{H}|=\frac{P}{A_{c s}}$, where $\boldsymbol{E}$ and $\boldsymbol{H}$ are the electric and magnetic fields, respectively [15]. The involvement of $\boldsymbol{E}$ and $\boldsymbol{H}$ triggers a spatiotemporal perturbation of the surface charge density, which can be described as $\sigma(\boldsymbol{r}, t)=\frac{q(\boldsymbol{r}, t)}{A_{c s}}$. To mathematically sketch such perturbation we hypothesize that, while hitting the surface of the 
capacitor, light affects the charges through a force $\boldsymbol{f}(t)=q(t) \boldsymbol{E}(t)$. Such force displaces the surface charges away from the location in which the visible or IR light impinges on the capacitor. Such a phenomenon is pictured for a broad range of low frequency electromagnetic (EM) waves in review articles (Ref. [34], page 11; Ref. [35], pages 7 and 13). Since the electric field $\boldsymbol{E}$ is related to the average power $P(t)=A_{c s}|\boldsymbol{S}(t)|=A_{c s}|\boldsymbol{E}(t) \times \boldsymbol{H}(t)|$, we can further picture that the surface charge density locally decreases such that $\sigma(\boldsymbol{r}, t) \propto \frac{1}{P(t)}$ without ejecting charges away of the capacitor's surface. To depict reference system, orientation and phase of the spatial variables defining $\sigma(\boldsymbol{r}, t)$, we represent the 2D space variable $r$ as a complex variable $z=r_{x}+i r_{y}$, where $i$ is the imaginary unit. This choice resembles that adopted to describe light polarization through Jones matrices [36] [37] [38]. Thus,

$$
\boldsymbol{z}=\left[\begin{array}{l}
r_{x} \\
r_{y}
\end{array}\right]=\left[\begin{array}{l}
r_{0 \mathrm{x}} \mathrm{e}^{i \phi_{x}} \\
r_{0 y} \mathrm{e}^{i \phi_{y}}
\end{array}\right]=\left[\begin{array}{l}
r_{a}+i r_{b} \\
r_{c}+i r_{d}
\end{array}\right] .
$$

All possible rotations of the reference system, phases, and positions in the $2 \mathrm{D}$ plane can be obtained by selecting magnitude and sign of $r_{a}, r_{b}, r_{c}$, and $r_{d}$. With this premise we can model $\sigma(z, t)$ as exponentially decreasing with the increase of $P(t)$ according to:

$$
\begin{aligned}
\sigma(\boldsymbol{z}, t) & =R E\left(\left|\sigma_{0}\right| \mathrm{e}^{-\frac{t}{\tau_{\sigma}}+r_{x} k_{x}+\varphi} \mathrm{e}^{i r_{y} k_{y}}-\left|\sigma_{f}\right|\right) . \\
& =\left|\sigma_{0}\right| \mathrm{e}^{-\frac{t}{\tau_{\sigma}}+f_{x} L_{x} k_{x}+\phi} \cos \left(f_{y} L_{y} k_{y}\right)-\left|\sigma_{f}\right|
\end{aligned}
$$

The parameters $\sigma_{0}$ and $\sigma_{f} \approx \sigma_{s s l i}$ are surface charge densities, where $\sigma_{s s l i}$ is the value in the ssli. They are considered as absolute values because the density of the charge is independent of the charge's sign. The time constant $\tau_{\sigma}$ of the surface charge density's decay is effective with a slight delay compared to the time constant $\tau_{P}$ of the power $P(t)$. $\boldsymbol{k}$, with components $k_{x}$ and $k_{y}$, is a vector with units of inverse length. To simplify the determination of the magnitude of the phase $\varphi$, of $k_{x}$ and $k_{y}$, we assume that $\left|k_{x}\right|=\left|k_{y}\right|=\kappa$. The quantities $L_{x}$ and $L_{y}$ are such that $\left|L_{x}\right|=\left|L_{y}\right|=D$. We note that the exponential behavior of $\sigma(z, t)$ is modulated by the oscillatory function $\mathrm{e}^{i r_{y} K}$. In Equation (A10) we write the spatial variables as follows: $r_{x}=f_{x} L_{x}$ and $r_{y}=f_{y} L_{y}$, where $f_{x}=\frac{r_{x}}{L_{x}}$ and $f_{y}=\frac{r_{y}}{L_{y}}$ are dimensionless fractions varying from 0 to 1 and enabling us to locate any position on the surface of the capacitor. For example, by choosing the origin of the reference systems on the lower left corner as in Figure $\mathrm{A} 3$, we have that when $r_{x}=0 \quad\left(r_{y}=0\right)$ then $f_{x}=0 \quad\left(f_{y}=0\right)$. When $r_{x}=D \quad\left(r_{y}=D\right)$ then $f_{x}=1 \quad\left(f_{y}=1\right)$. The dimensionless fractions will allow us to calculate the integral of $\sigma(\boldsymbol{r}, t)$ over space in sub-section A2-C. In Figure A4 we show a $3 \mathrm{D}$ representation of the evolution in time of the surface charge 


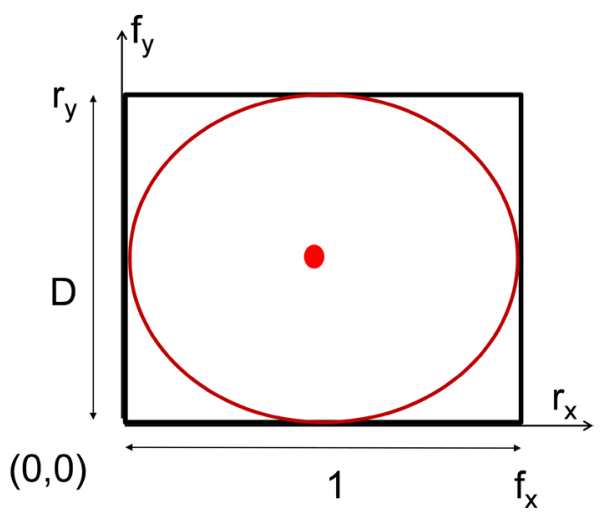

Figure A3. Description of the visible or IR laser's cross section with diameter $D$, centered in the thick dot and impinging on the surface of the capacitor. The origin of the reference systems of the spatial coordinates $\left(r_{x}, r_{y}\right)$ and of the dimensionless fractions $\left(f_{x}, f_{y}\right)$ coincide. The spatial coordinates $r_{x}$ and $r_{y}$ span from 0 to $D$, while the dimensionless fractions $f_{x}$ and $f_{y}$ vary from 0 to 1 .

(a) $5 \mathrm{~s}$ (b) $1000 \mathrm{~s}$

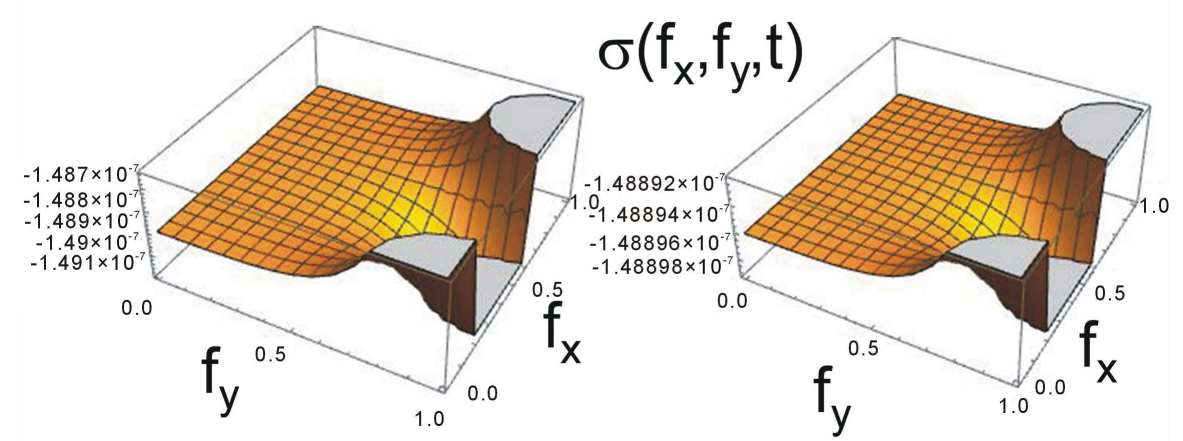

Figure A4. 3D representation of the evolution after $5 \mathrm{~s}$ and $1000 \mathrm{~s}$ after the beginning of the illumination of the surface charge density $\sigma\left(f_{x}, f_{y}, t\right)$ according to Equation (A10) and pertinent to the specific case of Figure 1 (a) with $C=43.4 \mathrm{pF}$, and illumination with a continuous wave (c.w.) laser with $P=700 \mathrm{~mW}, D=3 \mathrm{~mm}$, and $\tau=3.55 \mathrm{fs}$. The changes of the values on the z-axis for $\sigma\left(f_{x}, f_{y}, t\right)$ are as follows: after $5 \mathrm{~s}$ from $-1.491 \times 10^{-7} \frac{\mathrm{C}}{\mathrm{m}^{2}}$ to $-1.487 \times 10^{-7} \frac{\mathrm{C}}{\mathrm{m}^{2}}$, and after $1000 \mathrm{~s}$ from $-1.48898 \times 10^{-7} \frac{\mathrm{C}}{\mathrm{m}^{2}}$ to $-1.48892 \times 10^{-7} \frac{\mathrm{C}}{\mathrm{m}^{2}}$.

density according to Equation (A10) and pertinent to the specific case of Figure 1 (a).

A2-C. The integral of $\sigma(r, t)$ over space.

While $\sigma(z, t)$ is defined by a spatiotemporal set of variables, the functions $\Delta V(t)$ and $\Delta T(t)$ depend only on time $t$. To decouple the spatial variable $z$ 
from $\sigma(z, t)$, we integrate $\sigma(z, t)$ in Equation (A10) over the illuminated surface area of the capacitor approximated as a square with side $D$. We thus obtain a time-dependent surface charge density $\sigma(t)$ such that:

$$
\begin{aligned}
\sigma(t) & =-\left|\sigma_{f}\right|+\left|\sigma_{0}\right| \int_{A_{c s}} \mathrm{~d} A_{c s}\left(\mathrm{e}^{-\frac{t}{\tau_{\sigma}}+f_{x} L_{x} \kappa+\varphi} \cos \left(f_{y} L_{y} \kappa\right)\right) \\
& =-\left|\sigma_{f}\right|+\left|\sigma_{0}\right| \mathrm{e}^{-\frac{t}{\tau_{\sigma}}+\varphi} \int_{f_{x 0}}^{f_{x f}} \mathrm{~d} f_{x} \mathrm{e}^{f_{x} L_{x} \kappa} \int_{f_{y 0}}^{f_{y f}} \mathrm{~d} f_{y} \cos \left(f_{y} L_{y} \kappa\right) .
\end{aligned}
$$

To solve the integrals, we change the variables from $f_{x}$ to $\Xi=f_{x} L_{x} \kappa$, and from $f_{y}$ to $\mathrm{Y}=f_{y} L_{y} \kappa$. We obtain $\mathrm{d} f_{x}=\frac{\mathrm{d} \Xi}{L_{x} \kappa}, \quad \Xi_{0}=f_{x 0} L_{x} \kappa$, and $\Xi_{f}=f_{x f} L_{x} \kappa$. Similarly, we also have $\mathrm{d} f_{y}=\frac{\mathrm{dY}}{L_{y} \kappa}, \quad \mathrm{Y}_{0}=f_{y 0} L_{y} \kappa$, and $\mathrm{Y}_{f}=f_{y f} L_{y} \kappa$. Consequently, $\sigma(t)$ becomes:

$$
\begin{aligned}
\sigma(t) & =-\left|\sigma_{f}\right|+\left|\sigma_{0}\right|\left(\frac{\mathrm{e}^{-\frac{t}{\tau_{\sigma}}+\varphi}}{L_{x} L_{y} \kappa^{2}}\right) \int_{\Xi_{0}}^{\Xi_{f}} \mathrm{~d} \Xi e^{\Xi} \int_{\mathrm{Y}_{0}}^{\mathrm{Y}_{f}} \mathrm{~d} \mathrm{Y} \cos (\mathrm{Y}) \\
& =-\left|\sigma_{f}\right|+\left|\sigma_{0}\right|\left(\frac{\mathrm{e}^{-\frac{t}{\tau_{\sigma}}+\varphi}}{L_{x} L_{y} \kappa^{2}}\right)\left(e^{\Xi_{f}}-e^{\Xi_{0}}\right)\left(\sin \left(\mathrm{Y}_{f}\right)-\sin \left(\mathrm{Y}_{0}\right)\right) \\
& =-\left|\sigma_{f}\right|+\left|\sigma_{0}\right|\left(\frac{\mathrm{e}^{-\frac{t}{\tau_{\sigma}}+\varphi}}{L_{x} L_{y} \kappa^{2}}\right)\left(e^{f_{x x} L_{x} \kappa}-e^{f_{x 0} L_{x} \kappa}\right)\left(\sin \left(f_{y f} L_{y} \kappa\right)-\sin \left(f_{y 0} L_{y} \kappa\right)\right)
\end{aligned}
$$

To eliminate the $f_{x}$ and $f_{y}$ variables in Equation (A12) we choose the reference system for $f_{x}$ and $f_{y}$ as in Figure $\mathrm{A} 3$, so that $\left(f_{x 0}, f_{x f}\right)=(0,1)$ and $\left(f_{y 0}, f_{y f}\right)=(0,1)$. With such choice $\sigma(t)$ results in:

$$
\sigma(t)=-\left|\sigma_{f}\right|+\left|\sigma_{0}\right|\left(\frac{\mathrm{e}^{-\frac{t}{\tau_{\sigma}}+\varphi}}{L_{x} L_{y} \kappa^{2}}\right)\left(e^{L_{x} \kappa}-1\right) \sin \left(L_{y} \kappa\right) .
$$

From Equation (A13) we can infer that to evaluate $q(t)=\sigma(t) * A_{c s}$ requires knowing the values of $\sigma_{f}, \sigma_{0}, \tau_{\sigma}, \varphi$, and $\kappa$. A rough value for $\sigma_{f}$ can be estimated from Equation (A8b) such that:

$$
\sigma f \approx \frac{C \Delta V}{A_{c s}} .
$$

We evaluate the parameters $\tau_{\sigma}, \varphi$, and $\kappa$ from the best fit of the model to the experimental data for the voltage difference $\Delta V(t)$. We found the magnitude of $q(t)=\sigma(t) * A_{c s}$ in the $p C$ range in agreement with Ref. [39].

A2-D. Calculation of the correction factor $\zeta$.

In the EPR the visible and IR lasers significantly disturb the surface charge density $\sigma(t)$ on the capacitor through a sudden transition from a quiescent state to a perturbed one. A realistic view of the phenomenon requires going 
beyond the simple assumption that $\sigma(t)=\frac{\Delta V(t) C}{A_{c s}}$ adopted in Sub-section A2-A. Indeed, since both electrical and thermal phenomena are activated on the capacitor by the illumination through visible and IR lasers, we can assume that $\sigma(t)=\sigma(t)_{e l}+\sigma(t)_{t h}$. The electrical phenomena induce a voltage difference $\Delta V(t)_{e l}$ linked to the capacitive behavior of the capacitors with capacitance $\mathrm{C}$ through the surface charge density $\sigma(t)_{e l}$. We depict $\sigma(t)_{e l}$ as:

$$
\sigma(t)_{e l} \approx \frac{\Delta V(t) C}{A_{c s}}
$$

On the other hand, we assume that the thermal phenomena induce a voltage difference $\Delta V(t)_{t h}$ linked to the raise in temperature difference $\Delta T(t)$ associated with a surface charge density $\sigma(t)_{t h}$. Since it is challenging to directly relate the temperature difference $\Delta T(t)$ to $\sigma(t)_{t h}$, we assume $\sigma(t)_{t h}$ to be:

$$
\sigma(t)_{t h}=\frac{\zeta \Delta V(t)^{2}}{A_{c s}}
$$

where $\zeta$ is a correction factor related to $\Delta T(t)$.

With these observations, the overall surface charge density $\sigma(t)$ becomes:

$$
\sigma(t)=\sigma(t)_{e l}+\sigma(t)_{t h}=\frac{\Delta V(t) C+\zeta \Delta V(t)^{2}}{A_{c s}}
$$

and the correction factor $\zeta$ can be evaluated as:

$$
\zeta=\frac{q(t)}{\Delta V(t)^{2}}-\frac{C}{\Delta V(t)} .
$$

The magnitude of $\zeta$ has units of $\frac{\mathrm{nC}}{\mathrm{V}^{2}}$ and can be extracted from the experimental data. For each set of data obtained with the same type of laser, $\tau$, and $C$, we display $\zeta$ versus average power $P$ in Figure A5 and observe that $\zeta$ levels-off at a value which we call the equilibrium correction factor $\zeta_{e q}$. In Figure A6 we collect the values of $\zeta_{e q}$ versus $C$ and $\tau$, respectively, in the case of c.w. and PL lasers illuminating the capacitors with various values of the dielectric constant $\varepsilon$ reported in Table A1 and Table A2. The values of $\zeta_{e q}$ vary between about -100 and $100 \frac{\mathrm{nC}}{\mathrm{V}^{2}}$. As expected, we note that $\zeta_{\text {eq }}$ significantly deviates from the zero line for large $\Delta T$ as $\tau$ moves toward the visible region of the EM spectrum (see Figure 2(d) and Figure A7(d)). However, we also discover that large capacitances $C$ contribute to large deviations of $\zeta_{e q}$ from the zero line.

\section{Appendix-3. Additional Trends of the Voltage $\pi_{\Delta} v$ and Temperature $\zeta_{\Delta} T$ Responsivities with Capacitance $C$ and Laser's Period $\tau$}

In Figure $\mathrm{A} 7(\mathrm{a})$ and Figure $\mathrm{A} 7(\mathrm{~b})$ we plot the voltage $\pi_{\Delta V}$ and temperature 

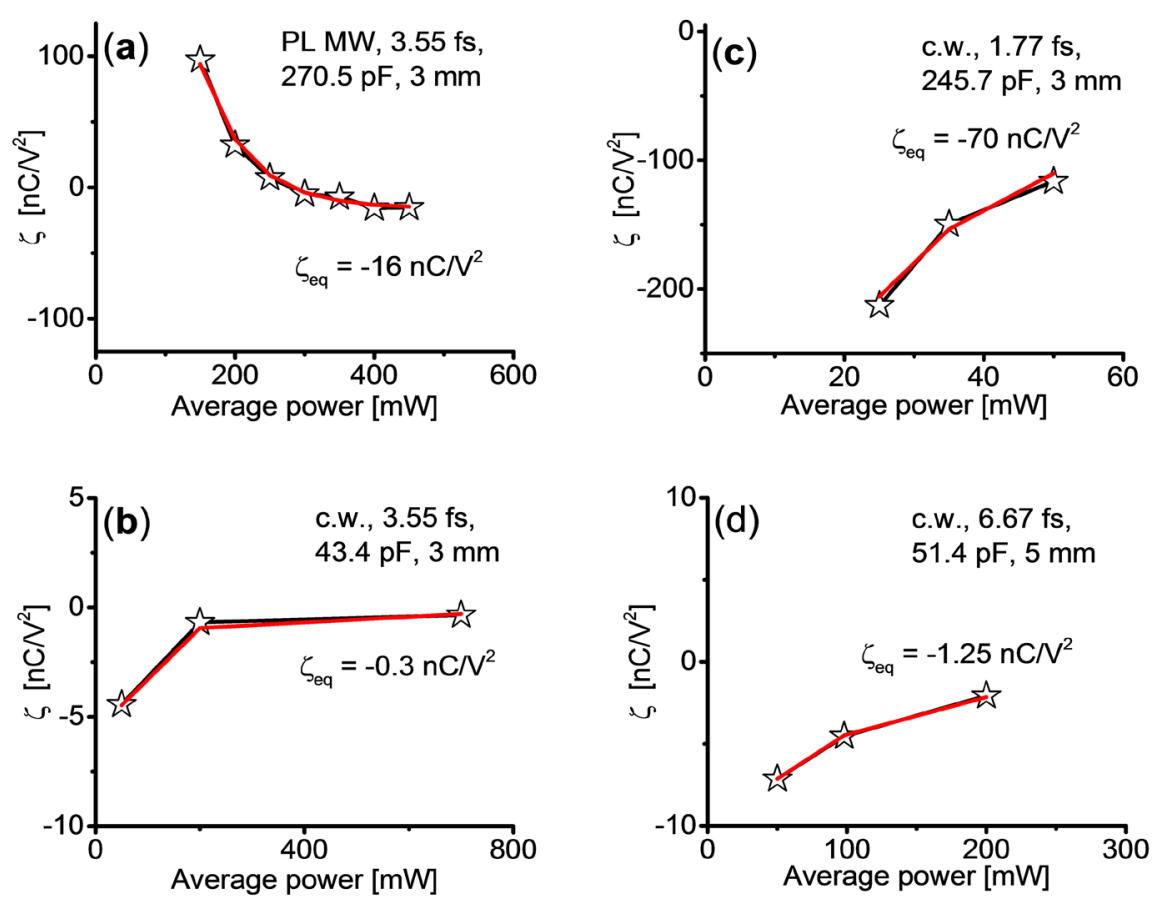

Figure A5. Correction factor $\zeta$ versus average power $P$ for the following cases: (a) pulsed laser (PL), $\tau=3.55 \mathrm{fs}, C=270.5 \mathrm{pF}, D=3 \mathrm{~mm}$. (b) continuous wave (c.w.) laser, $\tau$ $=3.55 \mathrm{fs}, C=43.4 \mathrm{pF}, D=3 \mathrm{~mm}$. (c) c.w., $\tau=1.77 \mathrm{fs}, C=245.7 \mathrm{pF}, D=3 \mathrm{~mm}$. (d) c.w., $\tau$ $=6.67 \mathrm{fs}, C=51.4 \mathrm{pF}, D=5 \mathrm{~mm}$. Each panel reports the value of the corresponding equilibrium correction factor $\zeta_{e q}$.
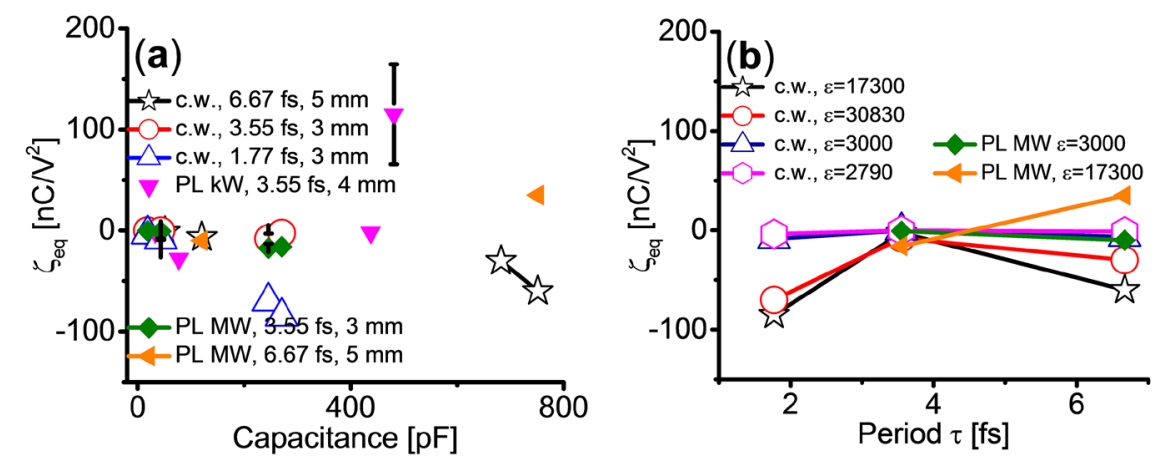

Figure A6. Equilibrium correction factor $\zeta_{e q}$ versus (a) capacitance $C$ and (b) period $\tau$ for the cases of continuous wave (c.w.) and pulsed (PL) lasers with various values of cross-sectional diameter $D$ illuminating capacitors with various values of the dielectric constant $\varepsilon$ reported in Table A1 and Table A2. The PL lasers we used to obtain the results with $\tau=6.67 \mathrm{fs}$ are deascribed in Table A3.

$\xi_{\Delta T}$ responsivities versus the capacitance $C$ of devices consisting of two capacitors in series. These devices are described in Figure A2 and their characteristics are summarized in Table A2. In Figure A7(a) and Figure A7(b) we observe that $\pi_{\Delta V}$ increases when $C$ decreases, while, within the uncertainties, $\xi_{\Delta T}$ is not sensitive to $C$. In Figure A7(c) and Figure A7(d) we report the trends of the $\pi_{\Delta V}$ and $\xi_{\Delta T}$ responsivities versus the period $\tau$ of the c.w. lasers obtained 

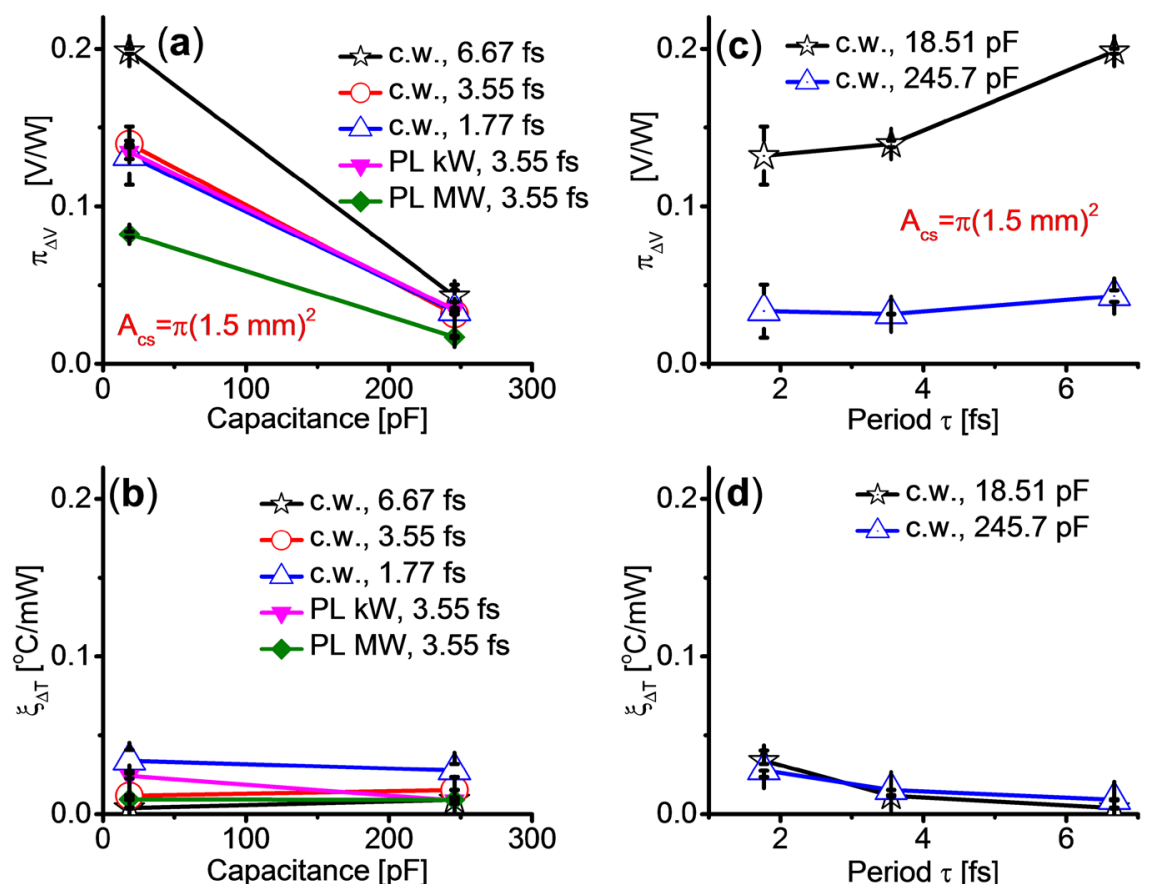

Figure A7. Trends of the (a) voltage $\pi_{\Delta V}$ and (b) temperature $\xi_{\Delta T}$ responsivities and corresponding uncertainties versus capacitance $C$ for capacitors illuminated on a cross-sectional area $A_{c s}$ with radius $\frac{D}{2}=1.5 \mathrm{~mm}$ with continuous wave (c.w.) lasers of periods $\tau=1.77 \mathrm{fs}(\lambda=532 \mathrm{~nm}), 3.55 \mathrm{fs}(\lambda=1064 \mathrm{~nm})$, and $6.67 \mathrm{fs}\left(\lambda=2 \times 10^{3} \mathrm{~nm}\right)$. We also display data obtained upon illumination of the same capacitors with pulsed (PL) lasers with $\tau=3.55 \mathrm{fs}$ and $P_{p e a k}$ in the kW and MW ranges. Trends of the (c) $\pi_{\Delta V}$ and (d) $\xi_{\Delta T}$ responsivities and corresponding uncertainties versus period $\tau$ of the visible and IR lasers. The data were collected with c.w. lasers with period $\tau=1.77 \mathrm{fs}, 3.55 \mathrm{fs}$, and $6.67 \mathrm{fs}$ illuminating a cross-sectional area $A_{c s}$ with radius $\frac{D}{2}=1.5 \mathrm{~mm}$ on capacitors with $C=$ $18.51 \mathrm{pF}$ and $C=245.7 \mathrm{pF}$. These capacitors are described in Figure A2 and their characteristics are summarized in Table A2.

Table A3. Mode of operation (monochromatic), period $\tau$, maker and type, repetition rate $\frac{1}{T_{p}}$, pulse duration $\Delta t_{p}$, average power $P$ range, peak power $P_{\text {peak }}$ range, and beam diameter $D$ of the IR pulsed laser (PL) sources used to collect the data with $\tau=6.67$ fs discussed in Figure A6. We used continuum surelite optical parametric oscillator (OPO) lasers to tune the desired beams to specified wavelengths. These lasers were pumped with a frequency-tripled continuum surelite-II laser.

\begin{tabular}{cccccccc}
\hline $\begin{array}{c}\text { Mode of } \\
\text { operation }\end{array}$ & $\begin{array}{c}\text { Period } \\
\tau[\mathrm{fs}]\end{array}$ & $\begin{array}{c}\text { Maker and } \\
\text { type }\end{array}$ & $\begin{array}{c}\text { Repetition } \\
\text { rate } 1 / \mathrm{T}_{\mathrm{p}} \\
{[1 / \mathrm{s}]}\end{array}$ & $\begin{array}{c}\text { Pulse } \\
\text { duration } \\
\Delta \mathrm{t}_{\mathrm{p}}[\mathrm{ns}]\end{array}$ & $\begin{array}{c}\text { Average } \\
\text { power P } \\
\text { range } \\
{[\mathrm{mW}]}\end{array}$ & $\begin{array}{c}\text { Peak } \\
\text { power } \\
\mathrm{P}_{\text {peak }} \\
\text { range }\end{array}$ & $\begin{array}{c}\text { Beam } \\
\text { diameter }\end{array}$ \\
\hline $\mathrm{PL}$ & 6.67 & $\begin{array}{c}\text { Thor- } \\
\text { Labs-OPO }\end{array}$ & 20 & 4 & $20-70$ & $\mathrm{MW}$ & 5 \\
\hline
\end{tabular}

${ }^{\star}$ Continuum surelite OPO laser tuned to specified wavelengths and pumped with frequency-tripled continuum surelite-II laser. 
using devices consisting of two capacitors in series with $C=18.5 \mathrm{pF}$ and $C=245.7 \mathrm{pF}$. We observe an increase in $\pi_{\Delta V}$ when $\tau$ increases, i.e. toward the far IR and microwave regions of the EM spectrum. The temperature responsivity $\xi_{\Delta T}$ exhibits a slight increase toward the visible region of the EM spectrum. The trends in Figure A7 are similar to those in Figure 2. The most notable difference between the two figures is that the uncertainties are more pronounced in Figure A7 than in Figure 2. 